\title{
Qualitative behavior of a Keller-Segel model with non-diffusive memory
}

\author{
Kyungkeun Kang*, Angela Stevens†, Juan J. L. Velázquez ${ }^{\ddagger}$
}

\begin{abstract}
In this paper a one-dimensional Keller-Segel model with a logarithmic chemotacticsensitivity and a non-diffusing chemical is classified with respect to its long time behavior. The strength of production of the non-diffusive chemical has a strong influence on the qualitative behavior of the system concerning existence of global solutions or Dirac-mass formation. Further, the initial data play a crucial role.
\end{abstract}

\section{Introduction}

We consider a chemotaxis-system with a logarithmic chemotactic sensitivity and a nondiffusing chemical. The main question addressed is whether smooth solutions exist globally in time, or blowup happens. A crucial assumption is that the chemical is produced by the chemotactic species and decay terms do not occur. Thus a drift-diffusion equation is coupled to an ODE. In [5] Keller and Segel discussed traveling waves for a similar system, where for the chemical reaction kinetics just a decay term is considered. Thus existence of global solutions can always be expected. When varying the strength of the production an interesting long time behavior can be expected for the system, as introduced in [11] and formally explored in [8]. Existence of global solutions for linear production kinetics with respect to the chemotactic species was proved in [13]. For a fixed and strong production kinetics in [6] finite time blowup was shown for specific explicit initial data.

In this paper we classify the system for a variety of production kinetics and types of initial data. The aim is to find "critical conditions" for the switch between existence of global solutions and Dirac mass formation.

The system we study is

$$
u_{t}=u_{x x}-\left(u \frac{w_{x}}{w}\right)_{x}, \quad w_{t}=u w^{\lambda} \quad \text { for } t>0, x \in I=[0, \pi], \text { and } \lambda \in[0,1),
$$

\footnotetext{
${ }^{*}$ Department of Mathematics, Sungkyunkwan University and Institute of Basic Science, Suwon 440-746, Republic of Korea (kkang@skku.edu)

${ }^{\dagger}$ University of Heidelberg, Applied Mathematics and Bioquant, BQ 0021, INF 267, D-69120 Heidelberg, Germany (angela.stevens@uni-hd.de)

${ }^{\ddagger}$ Departamento de Matemática Aplicada, Facultad de Ciencias Matemáticas, 28040 Madrid, Spain (JJ_Velazquez@mat.ucm.es)
} 
with periodic boundary conditions. Here $u$ models the chemotactic species and $w$ the non-diffusive memory. By setting $\theta=\frac{1}{1-\lambda}$ and $z=w^{\frac{1}{\theta}}$ we obtain

$$
u_{t}=u_{x x}-\theta\left(u \frac{z_{x}}{z}\right)_{x}, \quad z_{t}=u \quad \text { for } t>0, x \in I=[0, \pi] .
$$

So $\theta \in[1, \infty)$. In [13] a result for $\lambda=0$, respectively $\theta=1$ was obtained. In [6] a result for $\lambda=1$ was given. We will have a closer look at the regime in between, where the interesting switch from the existence of global solutions toward Dirac mass formation is to be expected.

Throughout this paper we will use the following notation for the functional spaces for given $t>0$ :

$$
H^{k}=H^{k}(I)=\left\{f(t, \cdot): D_{j} f(t, \cdot) \in L^{2}(I), 0 \leq j \leq k\right\} .
$$

\section{Qualitative behavior of system for $\theta=1$ :}

In this section $C$ will always denote a generic constant that can change from line to line. We will show that there exist global smooth solutions for system (1) with periodic boundary conditions. In [13] an $L^{\infty}$-estimate was proved for this case.

First note that $u(x, t)=a$ and $z(x, t)=a t+b$ with $a, b>0$ are homogeneous solutions of

$$
u_{t}=u_{x x}-\left(u \frac{z_{x}}{z}\right)_{x}, \quad z_{t}=u \quad \text { for } t>0, x \in I=[0, \pi] .
$$

For convenience, define $\bar{z}(t)=a t+b$. We will first study the stability for this problem. Due to translation, e.g. $\tau=t+\frac{b}{a}$, one can assume w.l.o.g. that $t \geq \frac{b}{a}$, so $\bar{z}(t)=a t$. For simplicity, we set $a=b$. Our main result of this section is

Theorem 2.1 Let (a,at) be a space-independent solution of (1), where $a>0$ is constant. If $(u, z)$ is a solution with initial data $\left(u_{0}, z_{0}\right)$ sufficiently close to $(a, a)$, then there exists $v_{\infty} \in H^{2}$ such that $u$ and $\frac{z}{t}$ both converge to $a+v_{\infty}$ for $t \rightarrow \infty$.

We need several steps to prove this theorem. So we are looking for solutions of type

$$
u(x, t)=a+v(x, t), \quad z(x, t)=\bar{z}(t)+\zeta(x, t) .
$$

Assume that $v_{0}(x)=v(x, 1)$ and $\zeta_{0}(x)=\zeta(x, 1)$ are "sufficiently" small and regular. Further details will be specified later. Substituting (2) into (1), we have

$$
v_{t}=v_{x x}-\left((a+v) \frac{\zeta_{x}}{\bar{z}+\zeta}\right)_{x}=v_{x x}-\left(\frac{1}{t} \zeta_{x}\right)_{x}-\left(\frac{\zeta_{x}}{\bar{z}+\zeta} v-\frac{a \zeta \zeta_{x}}{\bar{z}(\bar{z}+\zeta)}\right)_{x}, \quad \zeta_{t}=v .
$$

Also we will consider the Fourier-expansion

$$
v(x, t)=\sum_{n=-\infty}^{\infty} v_{n}(t) e^{i n x}, \quad \zeta(x, t)=\sum_{n=-\infty}^{\infty} \zeta_{n}(t) e^{i n x} .
$$




\subsection{The linearized problem}

First we linearize system (3) around the homogenous solutions and obtain

$$
v_{t}=v_{x x}-\frac{1}{t} \zeta_{x x}, \quad \zeta_{t}=v, \quad \text { in } I=[0, \pi] .
$$

We show that the space-independent solution $(a, a t)$ of $(3)$ is stable on the linearized level.

Proposition 2.2 Let $(v, \zeta)$ be a solution of (4). There exist $\epsilon>0$ and $\delta=\delta(\epsilon)$ such that for $\|v(1)\|_{H^{2}}+\|\zeta(1)\|_{H^{2}}<\delta$ one obtains $\|v(t)\|_{H^{2}} \leq \epsilon$ and $\|\zeta(t)\|_{H^{2}} \leq \epsilon t$ for all $1 \leq t<\infty$. Moreover, there exists $v_{\infty} \in H^{2}$ with

$$
\int_{\Omega} v_{\infty} e^{i n x} d x=\zeta_{n}(1)-\left(\zeta_{n}(1)-v_{n}(1)\right) \int_{1}^{\infty} \frac{e^{n^{2}(1-s)}}{s^{2}} d s
$$

such that

$$
\left\|v(t)-v_{\infty}\right\|_{H^{2}}, \quad\left\|\frac{\zeta(t)}{t}-v_{\infty}\right\|_{H^{2}} \longrightarrow 0 \quad \text { as } t \rightarrow \infty .
$$

Proof. The Fourier coefficients of $v$ and $\zeta$ must satisfy

$$
v_{n}^{\prime}(t)=-n^{2} v_{n}(t)+\frac{1}{t} n^{2} \zeta_{n}(t), \quad \zeta_{n}^{\prime}(t)=v_{n}(t) .
$$

Therefore,

$$
\zeta_{n}^{\prime \prime}(t)=-n^{2} \zeta_{n}^{\prime}(t)+\frac{1}{t} n^{2} \zeta_{n}(t)
$$

Solving this ODE, we get

$$
\zeta_{n}(t)=A_{n} t+B_{n} t \int_{t}^{\infty} \frac{e^{-n^{2} s}}{s^{2}} d s, \quad v_{n}(t)=A_{n}+B_{n} \int_{t}^{\infty} \frac{e^{-n^{2} s}}{s^{2}} d s-\frac{B_{n}}{t} e^{-n^{2} t}
$$

where

$$
A_{n}=\zeta_{n}(1)-\left(\zeta_{n}(1)-v_{n}(1)\right) \int_{1}^{\infty} \frac{e^{n^{2}(1-s)}}{s^{2}} d s, \quad B_{n}=e^{n^{2}}\left(\zeta_{n}(1)-v_{n}(1)\right) .
$$

Formula (5) is valid also for $n=0$, in which case $\zeta_{0}(t)=A_{0} t+B_{0}$ and $v_{0}(t)=A_{0}$. Due to the assumptions on the initial conditions, we have $\sum_{n=-\infty}^{\infty}\left(1+n^{4}\right)\left(\left|v_{n}(1)\right|^{2}+\left|\zeta_{n}(1)\right|^{2}\right)<\delta^{2}$, and for all $n \in \mathbb{Z}$ we have $v_{n}-\zeta_{n} / t=$ $-B_{n} e^{-n^{2} t} / t$. Thus, direct computations show that

$$
\begin{gathered}
\left\|v-\frac{\zeta}{t}\right\|_{H^{2}}^{2} \leq \sum_{n=-\infty}^{\infty}\left(1+n^{4}\right) B_{n}^{2} \frac{e^{-2 n^{2} t}}{t^{2}}=\sum_{n=-\infty}^{\infty}\left(1+n^{4}\right)\left|\zeta_{n}(1)-v_{n}(1)\right|^{2} \frac{e^{2 n^{2}(1-t)}}{t^{2}} \\
\leq \frac{\|\zeta(1)-v(1)\|_{H^{2}}^{2}}{t^{2}}<\frac{\delta^{2}}{t^{2}}
\end{gathered}
$$


It is straightforward that

$$
\sum_{n=-\infty}^{\infty}\left(1+n^{4}\right) A_{n}^{2} \leq C \sum_{n=-\infty}^{\infty}\left(1+n^{4}\right)\left(\left|v_{n}(1)\right|^{2}+\left|\zeta_{n}(1)\right|^{2}\right) \leq C \delta^{2}
$$

and for any $t$ with $1 \leq t<\infty$ we have

$$
\begin{gathered}
\sum_{n=-\infty}^{\infty}\left(1+n^{4}\right) B_{n}^{2}\left(\int_{t}^{\infty} \frac{e^{-n^{2} s}}{s^{2}} d s\right)^{2} \leq \sum_{n=-\infty}^{\infty}\left(1+n^{4}\right)\left(\zeta_{n}(1)-v_{n}(1)\right)^{2}\left(\int_{t}^{\infty} \frac{e^{n^{2}(1-s)}}{s^{2}} d s\right)^{2} \\
\leq \sum_{n=-\infty}^{\infty}\left(1+n^{4}\right)\left(\zeta_{n}(1)-v_{n}(1)\right)^{2} \frac{1}{t^{2}}<\frac{\delta^{2}}{t^{2}}
\end{gathered}
$$

Summing up all estimates, we obtain

$$
\|v\|_{H^{2}}^{2} \leq \sum_{n=-\infty}^{\infty}\left(1+n^{4}\right)\left[A_{n}^{2}+B_{n}^{2}\left(\int_{t}^{\infty} \frac{e^{-n^{2} s}}{s^{2}} d s\right)^{2}+B_{n}^{2} \frac{e^{-2 n^{2} t}}{t^{2}}\right] \leq C \delta^{2}, \quad 1 \leq t<\infty
$$

and

$$
\left\|v-v_{\infty}\right\|_{H^{2}}^{2}=\sum_{n=-\infty}^{\infty}\left(1+n^{4}\right)\left[B_{n}^{2}\left(\int_{t}^{\infty} \frac{e^{-n^{2} s}}{s^{2}} d s\right)^{2}+B_{n}^{2} \frac{e^{-2 n^{2} t}}{t^{2}}\right] \leq C \frac{\delta^{2}}{t^{2}}
$$

This completes the proof.

\subsection{Nonlinear stability}

Let $f(x, t)$ be the nonlinear part of $(3)$ and let $f_{n}$ denote the n-th Fourier coefficient of $f$, namely

$$
f(x, t)=-\left(\frac{\zeta_{x}}{\bar{z}+\zeta} v-\frac{a \zeta \zeta_{x}}{\bar{z}(\bar{z}+\zeta)}\right)_{x}=-\left(\frac{\zeta_{x}}{\bar{z}+\zeta}\left(v-\frac{\zeta}{t}\right)\right)_{x}, \quad f_{n}(t):=\left(f(x, t), e^{i n x}\right) .
$$

Recalling (3) and comparing Fourier coefficients, we have

$$
\zeta_{n}^{\prime \prime}(t)+n^{2} \zeta_{n}^{\prime}(t)-\frac{1}{t} n^{2} \zeta_{n}(t)=f_{n}(t)
$$

By setting $\zeta_{n}(t)=t \Phi_{n}(t)$, we get

$$
\Phi_{n}^{\prime}(t)=\frac{e^{-n^{2} t}}{t^{2}} \int_{1}^{t} f_{n}(s) s e^{n^{2} s} d s . \quad \text { So }, \quad \Phi_{n}(t)=\int_{1}^{t} \frac{e^{-n^{2} \xi}}{\xi^{2}}\left(\int_{1}^{\xi} f_{n}(s) s e^{n^{2} s} d s\right) d \xi .
$$


The general solution of (6) is given by

$$
\begin{gathered}
\zeta_{n}(t)=A_{n} t+B_{n} t \int_{t}^{\infty} \frac{e^{-n^{2} s}}{s^{2}} d s+t \int_{1}^{t} \frac{e^{-n^{2} \xi}}{\xi^{2}}\left(\int_{1}^{\xi} f_{n}(s) s e^{n^{2} s} d s\right) d \xi \\
v_{n}(t)=A_{n}+B_{n} \int_{t}^{\infty} \frac{e^{-n^{2} s}}{s^{2}} d s-\frac{B_{n}}{t} e^{-n^{2} t}+\int_{1}^{t} \frac{e^{-n^{2} \xi}}{\xi^{2}}\left(\int_{1}^{\xi} f_{n}(s) s e^{n^{2} s} d s\right) d \xi+\frac{e^{-n^{2} t}}{t}\left(\int_{1}^{t} f_{n}(s) s e^{n^{2} s} d s\right),
\end{gathered}
$$

where

$$
A_{n}=\zeta_{n}(1)-B_{n} \int_{1}^{\infty} \frac{e^{-n^{2} s}}{s^{2}} d s, \quad B_{n}=e^{n^{2}}\left(\zeta_{n}(1)-v_{n}(1)\right) .
$$

In the sequel we assume that the solutions $\zeta(x, t)$ and $v(x, t)$ are in $H^{2}$, and that the initial data are small, i.e.

$$
\sum_{n=-\infty}^{\infty}\left(1+n^{4}\right)\left(\left|v_{n}(1)\right|^{2}+\left|\zeta_{n}(1)\right|^{2}\right)<\epsilon .
$$

Next we introduce the norm

$$
\||\psi|\|_{L, k}^{2}=\int_{(L-1)^{+}}^{L}\|\psi(t)\|_{H^{k}(\Omega)}^{2} d t, \quad k \geq 0,
$$

with $L>1,(L-1)^{+}=\max \{L-1,1\}$.

Lemma 2.3 Let $\psi$ be smooth in $\left[(L-1)^{+}, L\right] \times \Omega$. Then

$$
\sup _{(L-1)^{+}<t<L}\|\psi(t)\|_{H^{2}} \leq C\left(\|\| \psi\|\|_{L, 2}+\|\| \psi_{t} \mid \|_{L, 2}\right) .
$$

Proof. We note first that there exists $\bar{t} \in\left((L-1)^{+}, L\right)$ such that $\|\psi(\bar{t})\|_{H^{2}} \leq\|\| \psi \mid \|_{L, 2}$. Thus

$$
\psi(x, t)-\psi(x, \bar{t})=\int_{\bar{t}}^{t} \psi_{s}(x, s) d s, \quad \text { for any }(L-1)_{+}<t<L .
$$

Taking the $H^{2}$-norm on both sides of the equality and using Hölder's inequality, (7) is immediate.

Now we state local existence of small solutions.

Proposition 2.4 Let $(v, \zeta)$ be a solution of (3). There exist $\epsilon>0$ and $\delta=\delta(\epsilon)$ such that for $\|v(1)\|_{H^{2}}+\|\zeta(1)\|_{H^{2}}<\delta$ there exists $T=T(\epsilon)>0$ such that

$$
\sup _{L<T}\|\| v\left|\left\|\left.\right|_{L, 2}<\epsilon, \quad \sup _{L<T} \mid\right\| \zeta \|_{L, 2}<\epsilon L .\right.
$$

Proof. The proof is standard, so details are skipped. 
Lemma 2.5 Let $T \in(1, \infty]$ be the time in Proposition 2.4. Then for any $t<T$

$$
\|\zeta(t)\|_{L^{\infty}}+\|\zeta(t)\|_{H^{2}} \leq C \epsilon t .
$$

Proof. Since $\|\zeta(t)\|_{L^{\infty}} \leq C\|\zeta(t)\|_{H^{2}}$, it is sufficient to estimate $\|\zeta(t)\|_{H^{2}}$. Let $t \in$ $\left((L-1)^{+}, L\right)$ where $L<\min \{T, 2 t\}$. Due to Lemma 2.3 and Proposition 2.4 we have

$$
\|\zeta(t)\|_{H^{2}} \leq C\left(\left.|||\zeta|\right|_{L, 2}+\||| \zeta_{t}||_{L, 2}\right) \leq C\left(\left\|\left.|\zeta|\right|_{L, 2}+\right\||| v||_{L, 2}\right) \leq C \epsilon L \leq C \epsilon t .
$$

This completes the proof.

Lemma 2.6 Let $T \in(1, \infty]$ be the time in Proposition 2.4. Then for any $t<T$,

$$
\left\|v(t)-\frac{\zeta(t)}{t}\right\|_{H^{1}} \leq C \epsilon e^{-C t}, \quad\|f(t)\|_{L^{2}} \leq C \epsilon^{2} e^{-C t} .
$$

Proof. With (8) we can estimate $f(x, t)$ for $t \in[1, T)$ as follows

$$
\begin{gathered}
\|f(t)\|_{L^{2}(\Omega)} \leq\left\|\left(\frac{\zeta_{x}}{\bar{z}+\zeta}\left(v-\frac{\zeta}{t}\right)\right)_{x}\right\|_{L^{2}(\Omega)} \leq C\left(\frac{1}{t}\|\zeta\|_{H^{2}}\left\|v-\frac{\zeta}{t}\right\|_{H^{1}}+\frac{1}{t^{2}}\|\zeta\|_{H^{2}}^{2}\left\|v-\frac{\zeta}{t}\right\|_{L^{2}}\right) \\
\leq C\left(\epsilon+\epsilon^{2}\right)\left\|v-\frac{\zeta}{t}\right\|_{H^{1}} \leq C \epsilon\left\|v-\frac{\zeta}{t}\right\|_{H^{2}} .
\end{gathered}
$$

Since $v_{n}^{\prime}+n^{2} v_{n}-\frac{n^{2}}{t} \xi_{n}=f_{n}$, we have

$$
\frac{d}{d t}\left(v_{n}-\frac{\xi_{n}}{t}\right)+n^{2}\left(v_{n}-\frac{\xi_{n}}{t}\right)+\frac{1}{t}\left(v_{n}-\frac{\xi_{n}}{t}\right)=f_{n} .
$$

Multiplying with $n^{2}\left(v_{n}-\frac{\xi_{n}}{t}\right)$, we get

$$
\frac{n^{2}}{2} \frac{d}{d t}\left(v_{n}-\frac{\xi_{n}}{t}\right)^{2}+n^{4}\left(v_{n}-\frac{\xi_{n}}{t}\right)^{2}+\frac{n^{2}}{t}\left(v_{n}-\frac{\xi_{n}}{t}\right)^{2}=f_{n} n^{2}\left(v_{n}-\frac{\xi_{n}}{t}\right) .
$$

This implies that

$$
\frac{1}{2} \frac{d}{d t}\left\|v-\frac{\zeta}{t}\right\|_{H^{1}}^{2}+\left\|v-\frac{\zeta}{t}\right\|_{H^{2}}^{2}+\left\|\frac{1}{\sqrt{t}}\left(v-\frac{\zeta}{t}\right)\right\|_{H^{1}}^{2} \leq\|f\|_{L^{2}}\left\|v-\frac{\zeta}{t}\right\|_{H^{2}} .
$$

Due to (9), we obtain

$$
\frac{1}{2} \frac{d}{d t}\left\|v-\frac{\zeta}{t}\right\|_{H^{1}}^{2}+(1-C \epsilon)\left\|v-\frac{\zeta}{t}\right\|_{H^{2}}^{2} \leq 0 .
$$

Hence as long as $\epsilon$ is sufficiently small, by integrating over $[1, t)$ we obtain

$$
\left\|v(t)-\frac{\zeta(t)}{t}\right\|_{H^{1}} \leq\left\|v_{0}-\zeta_{0}\right\|_{H^{1}} e^{-C t} \leq \epsilon e^{-C t} .
$$

The second estimate is direct from (9) and (11). This completes the proof. 
Proposition 2.7 There exist $\epsilon>0$ and $\delta=\delta(\epsilon)$ such that for $\|v(1)\|_{H^{2}}+\|\zeta(1)\|_{H^{2}}<\delta$ we have \|\|$v\|\|_{t, 2} \leq \epsilon$ and $\|\mid \zeta\|_{t, 2} \leq \epsilon t$ for all $t<\infty$. Moreover, there exists $v_{\infty} \in H^{2}$ such that

$$
\begin{gathered}
\left\|\frac{\zeta(t)}{t}-v_{\infty}\right\|_{H^{2}} \longrightarrow 0 \quad \text { for } t \rightarrow \infty, \\
\left\|v(t)-v_{\infty}\right\|_{H^{1}} \longrightarrow 0 \quad \text { for } t \rightarrow \infty, \text { and } \\
\left\|v-v_{\infty}\right\|_{L, 2} \longrightarrow 0 \quad \text { for } L \rightarrow \infty .
\end{gathered}
$$

Proof. Let $T$ be the time in Proposition 2.4. We claim that $T=\infty$. Suppose that this is not the case, i.e. $T<\infty$. Then either $\||v|\|_{T, 2}>\epsilon$ or $\||\zeta|\|_{T, 2}>\epsilon T$. Suppose that $\||| v\|_{T, 2}>\epsilon$. For the case $\|\left.|\zeta|\right|_{T, 2}>\epsilon T$ we could argue similarly.

Recall the representation formula for $v_{n}$ :

$$
\begin{gathered}
v_{n}(t)=A_{n}+B_{n} \int_{t}^{\infty} \frac{e^{-n^{2} s}}{s^{2}} d s-\frac{B_{n}}{t} e^{-n^{2} t} \\
+\int_{1}^{t} \frac{e^{-n^{2} \xi}}{\xi^{2}}\left(\int_{1}^{\xi} f_{n}(s) s e^{n^{2} s} d s\right) d \xi+\frac{e^{-n^{2} t}}{t}\left(\int_{1}^{t} f_{n}(s) s e^{n^{2} s} d s\right),
\end{gathered}
$$

where

$$
A_{n}=\zeta_{n}(1)-B_{n} \int_{1}^{\infty} \frac{e^{-n^{2} s}}{s^{2}} d s, \quad B_{n}=e^{n^{2}}\left(\zeta_{n}(1)-v_{n}(1)\right) .
$$

It is sufficient to consider the nonlinear parts (12). For simplicity denote

$$
\Psi_{n}(t):=\int_{1}^{t} \frac{e^{-n^{2} \xi}}{\xi^{2}}\left(\int_{1}^{\xi} f_{n}(s) s e^{n^{2} s} d s\right) d \xi+\frac{e^{-n^{2} t}}{t}\left(\int_{1}^{t} f_{n}(s) s e^{n^{2} s} d s\right)=I_{n}(t)+I I_{n}(t) .
$$

Integrating by parts we obtain

$$
I_{n}(t)=-\frac{e^{-n^{2} t}}{n^{2} t^{2}} \int_{1}^{t} f_{n}(s) s e^{n^{2} s} d s+\frac{1}{n^{2}} \int_{1}^{t} \frac{f_{n}(\xi)}{\xi} d \xi-\frac{2}{n^{2}} \int_{1}^{t} \frac{e^{-n^{2} \xi}}{\xi^{3}}\left(\int_{1}^{\xi} f_{n}(s) s e^{n^{2} s} d s\right) d \xi .
$$

Direct computations show that

$$
\int_{(T-1)^{+}}^{T} \sum_{n=-\infty}^{\infty} n^{4} I_{n}^{2}(\tau) d \tau \leq C \int_{1}^{T} \sum_{n=-\infty}^{\infty} f_{n}^{2}(s) d s=C\|f\|_{L^{2}\left(Q_{T}\right)}^{2} \leq C \epsilon^{4} \int_{1}^{T} e^{-c t} d t \leq C \epsilon^{4} .
$$

Next consider

$$
\left|I I_{n}(t)\right| \leq \frac{e^{-n^{2} t}}{t}\left(\int_{1}^{t}\left|f_{n}(s)\right| s e^{n^{2} s} d s\right) \leq \int_{1}^{t}\left|f_{n}(s)\right| e^{n^{2}(s-t)} d s=: y(t) .
$$

Since the right hand side is a solution of $y^{\prime}(t)+n^{2} y(t)=\left|f_{n}\right|$, one can estimate

$$
\int_{(T-1)^{+}}^{T} \sum_{n=-\infty}^{\infty} n^{4} I I_{n}^{2}(\tau) d \tau \leq C \int_{1}^{T} \sum_{n=-\infty}^{\infty} f_{n}^{2}(s) d s=C\|f\|_{L^{2}\left(Q_{T}\right)}^{2} \leq C \epsilon^{4} .
$$


This can be seen by defining $y_{n}(t)=\int_{1}^{t}\left|f_{n}(s)\right| e^{n^{2}(s-t)} d s$. Thus $y_{n}^{\prime}(t)+n^{2} y_{n}(t)=\left|f_{n}(t)\right|$.

Define

$$
Y(x, t)=\sum_{n \neq 0} y_{n}(t) e^{i n x}, \quad F(x, t)=\sum_{n \neq 0}\left|f_{n}(t)\right| e^{i n x} .
$$

Then for $t \geq 1, Y$ solves in $I=[0, \pi]$ the following equation

$$
Y_{t}=Y_{x x}+F, \quad Y(x, 1)=0 .
$$

Further, classical estimates, using the fact that we do not have a neutral eigenvalue, yield

$$
\|Y(\cdot, t)\|_{L^{2}}^{2} \leq C \int_{1}^{T}\|F(\cdot, t)\|_{L^{2}}^{2} d t
$$

With classical regularity theory for the heat equation we obtain

$$
\begin{aligned}
\int_{(T-1)_{+}}^{T}\|Y(\cdot, t)\|_{H^{2}}^{2} & \leq C \sup _{t-1 \leq s \leq t}\|Y(\cdot, s)\|_{L^{2}}^{2}+C \int_{(T-1)_{+}}^{T}\|F(\cdot, t)\|_{L^{2}}^{2} d t \\
& \leq C \int_{1}^{T}\|F(\cdot, t)\|_{L^{2}}^{2} d t .
\end{aligned}
$$

Since

$$
\sum_{n} n^{4} I I_{n}^{2}=\sum_{n \neq 0} n^{4}\left|y_{n}(t)\right|^{2}=\|Y(\cdot, t)\|_{H^{2}}^{2},
$$

the estimate (13) follows. Summing up, we obtain

$$
\begin{gathered}
\|v\|_{T, 2} \leq\{\text { linear terms }\}+\left(\int_{(T-1)^{+}}^{T} \sum_{n=-\infty}^{\infty} n^{4} I_{n}^{2}(\tau) d \tau\right)^{\frac{1}{2}}+\left(\int_{(T-1)^{+}}^{T} \sum_{n=-\infty}^{\infty} n^{4} I I_{n}^{2}(\tau) d \tau\right)^{\frac{1}{2}} \\
\leq C \delta+C \epsilon^{2} .
\end{gathered}
$$

This shows $\||v|\|_{T, 2}<\epsilon$, which contradicts our hypothesis. Thus, $T$ cannot be finite.

Next we show convergence. We will prove that $I_{n}(t)=\zeta_{n} / t-A_{n} \in H^{2}$ and $I_{n}(t) \leq C \epsilon^{2}$ for all $t$. By changing the order of integration and using Hölder's inequality, we obtain

$$
\begin{gathered}
\left|I_{n}(t)\right|=\left|\int_{1}^{t} f_{n}(s) s e^{n^{2} s} \int_{s}^{t} \frac{e^{-n^{2} \xi}}{\xi^{2}} d \xi d s\right| \leq \int_{1}^{t}\left|f_{n}(s)\right| s e^{n^{2} s} \int_{s}^{t} \frac{e^{-n^{2} \xi}}{\xi^{2}} d \xi d s \\
\leq \frac{C}{n^{2}} \int_{1}^{t} \frac{\left|f_{n}(s)\right|}{s} d s \leq \frac{C}{n^{2}}\left(\int_{1}^{t}\left|f_{n}\right|^{2} d s\right)^{\frac{1}{2}}\left(\int_{1}^{t} s^{-2} d s\right)^{\frac{1}{2}} \leq \frac{C}{n^{2}}\left(\int_{1}^{t}\left|f_{n}\right|^{2} d s\right)^{\frac{1}{2}} .
\end{gathered}
$$

Due to Lemma 2.6, we have that $n^{4}\left|I_{n}(t)\right|^{2} \leq C \epsilon^{4}$ for all $t$. This implies that $\zeta / t$ is in $H^{2}$ and converges to $v_{\infty} \in H^{2}$ for $t \rightarrow \infty$, with

$$
v_{\infty}=\sum\left(v_{\infty}\right)_{n} e^{i n x}, \quad \text { where } \quad\left(v_{\infty}\right)_{n}=A_{n}+I_{n}(\infty),
$$


and

$$
I_{n}(\infty)=\int_{1}^{\infty} \frac{e^{-n^{2} \xi}}{\xi^{2}}\left(\int_{1}^{\xi} f_{n}(s) s e^{n^{2} s} d s\right) d \xi .
$$

Now it is direct that

$$
\frac{\zeta}{t} \longrightarrow v_{\infty} \quad \text { in } H^{2} \quad \text { for } t \rightarrow \infty
$$

From (10), we have

$$
\left\|v(t)-\frac{\zeta(t)}{t}\right\|_{H^{1}} \longrightarrow 0 \text { for } t \rightarrow \infty, \quad\|\| v(t)-\frac{\zeta(t)}{t}\|\|_{L, 2} \longrightarrow 0 \text { for } L \rightarrow \infty .
$$

Combining (14) and (15), we obtain that $v$ converges to $v_{\infty}$ in the $H^{1}$ and the $(L, 2)-$ norm, since

$$
\begin{gathered}
\left\|v-v_{\infty}\right\|_{H^{1}}+\left.\left\|v-v_{\infty}\right\|\right|_{L, 2} \leq\left\|v-\frac{\zeta}{t}\right\|_{H^{1}}+\left\|\frac{\zeta}{t}-v_{\infty}\right\|_{H^{1}}+\left\|v-\frac{\zeta}{t}\right\|\left\|_{L, 2}+\right\||| \frac{\zeta}{t}-v_{\infty}\|\|_{L, 2} \\
\leq\left\|v-\frac{\zeta}{t}\right\|_{H^{1}}+\|\| v-\frac{\zeta}{t}\|\|_{L, 2}+\left\|\frac{\zeta}{t}-v_{\infty}\right\|_{H^{2}} .
\end{gathered}
$$

This completes the proof.

Summarizing the previous estimates, for $\theta=1$ we obtain our main result of this section, namely Theorem 2.1.

\section{Qualitative behavior of the system for $1<\theta<3$}

From now on let $I=[-1,1]$. The reason for this change of domain of integration is simply to fix the expected singularity at the origin and avoid dealing with complicated shifts of its location. We consider

$$
\begin{gathered}
u_{t}=u_{x x}-\theta\left(\frac{z_{x}}{z} u\right)_{x}, \quad z_{t}=u \quad \text { in } I \times[0, \infty), \\
u(x, 0)=u_{0}(x), \quad z(x, 0)=z_{0}(x), \text { with periodic boundary conditions. }
\end{gathered}
$$

First, to get a quick insight, we give a heuristic argument regarding the blow-up asymptotics for this system for $t \rightarrow \infty$. After this we will go into the details of the rigorous analysis. For the heuristics we assume w.l.o.g. that $\int_{I} u d x=1$ and consider the simplified equation

$$
\bar{z}_{t}=\frac{\bar{z}^{\theta}}{\int_{I} \bar{z}^{\theta} d x} \text {. }
$$

We expect this simplified equation to be a good approximation for the dynamics of the original problem for $t \rightarrow \infty$. Assuming that $z_{0}(0)>z_{0}(x)$ for any $x \in I \backslash\{0\}$ we can solve this equation and obtain

$$
\bar{z}^{1-\theta}(x, t)=\bar{z}_{0}^{1-\theta}(x)-(\theta-1) \int_{0}^{t} \frac{d s}{\int_{I} \bar{z}^{\theta}(x, s) d x} .
$$


We assume further that $z_{0}$ can be expanded near zero as follows:

$$
\bar{z}_{0}^{1-\theta}(x)=\bar{z}_{0}^{1-\theta}(0)+B x^{2}+\text { h.o.t. } \quad \text { for } x \rightarrow 0 .
$$

Here $B$ is a positive constant depending on the initial data. Continuing the heuristic argument, we thus obtain

$$
\bar{z}^{1-\theta}(x, t) \approx \bar{z}_{0}^{1-\theta}(0)+B x^{2}-(\theta-1) \int_{0}^{t} \frac{d s}{\int_{I} \bar{z}^{\theta}(x, s) d x} .
$$

Define

$$
\psi(t):=\bar{z}_{0}^{1-\theta}(0)-(\theta-1) \int_{0}^{t} \frac{d s}{\int_{I} \bar{z}^{\theta}(x, s) d x} .
$$

Thus $\bar{z}^{1-\theta}(x, t) \approx B x^{2}+\psi(t)$, and

$$
\bar{z}(x, t) \approx \frac{1}{\left(B x^{2}+\psi(t)\right)^{\frac{1}{\theta-1}}} .
$$

Direct computations show

$$
-\frac{\theta-1}{\psi^{\prime}(t)} \approx \int_{I} \frac{d x}{\left(B x^{2}+\psi(t)\right)^{\frac{\theta}{\theta-1}}} .
$$

So we get $\psi^{\prime}(t) \approx-K \psi^{\frac{\theta+1}{2(\theta-1)}}(t)$, where $K$ is a positive constant. This yields $\psi(t) \approx$ $A t^{-\frac{2(\theta-1)}{3-\theta}}$ with a constant $A>0$ for $t \rightarrow \infty$. Since $\psi(t) \rightarrow 0$ for $t \rightarrow \infty$, we see that

$$
\bar{z}_{0}^{1-\theta}(0) \approx(\theta-1) \int_{0}^{\infty} \frac{d s}{\int_{I} \bar{z}^{\theta}(x, s) d x} .
$$

Therefore, noting that $\psi^{\prime}(t) \approx-K A^{\frac{\theta+1}{2(\theta-1)}} t^{-\frac{\theta+1}{3-\theta}}$ for $t \rightarrow \infty$, we obtain

$$
\psi(t) \approx(\theta-1) \int_{t}^{\infty} \frac{d s}{\int_{I} \bar{z}^{\theta} d x} \Longrightarrow \int_{I} \bar{z}^{\theta} d x \approx \frac{\theta-1}{K A^{\frac{\theta+1}{2(\theta-1)}}} t^{\frac{\theta+1}{3-\theta}},
$$

and

$$
\bar{z}(x, t) \approx \frac{1}{\left(B x^{2}+A t^{-\frac{2(\theta-1)}{3-\theta}}\right)^{\frac{1}{\theta-1}}}=\frac{t^{\frac{2}{3-\theta}}}{\left(B x^{2} t^{\frac{2(\theta-1)}{3-\theta}}+A\right)^{\frac{1}{\theta-1}}} .
$$

Now we are ready to present rigorous arguments which justify the given heuristics. A first idea for a quasi-steady state approximation of the system under consideration in the given regime for $\theta$ was given by Schwetlick, [10]. The main theorem we will prove in this section is the following 
Theorem 3.1 There exist initial data $u_{0}, z_{0} \in C^{2, \nu}$ such that the corresponding solutions $(u, z)$ of $(1)$ satisfy $u(x, t) \rightarrow m \delta(x)$ and $z(x, t) \approx \frac{t^{\frac{2}{3-\theta}}}{\left(B x^{2} t^{\frac{2(\theta-1)}{3-\theta}}+A\right)^{\frac{1}{\theta-1}}}$ for $t \rightarrow \infty$, where $m=\int_{I} u_{0}(x) d x$ and $A, B$ are constants depending on the initial data.

Remark: As we will see in Assumption 3.5 later, the condition on the initial data is, that $u_{0}, z_{0}$ are symmetric, $u_{0}$ is concentrated at the origin, and $z_{0}$ behaves like a power law at the origin. For convenience we will also assume in the following that $m=1$.

To prove this theorem we need several steps.

\subsection{The Eigenvalue problem}

We define the differential operator

$$
\tilde{A}_{z}(f):=f_{x x}-\theta\left(\frac{z_{x}}{z} f\right)_{x}=\left(f_{x}-\theta\left(\frac{z_{x}}{z} f\right)\right)_{x} \quad \text { in }[-1,1] .
$$

Consider the eigenvalue problem $\tilde{A}_{z}(f)=\lambda f$, i.e.

$$
f_{x x}-\theta\left(\frac{z_{x}}{z} f\right)_{x}=\lambda f, \quad f(-1)=f(1), f_{x}(-1)=f_{x}(1) .
$$

Since we have assumed periodic boundary conditions in $\mathbb{R}$, it is direct that $f(-1)=f(1)=$ $f_{x}(-1)=f_{x}(1)=0$. Now a class of functions $\mathcal{A}$ is introduced, which is assumed to contain $z$.

Assumption 3.2 Let $0<\nu<1$ and let $\mathcal{A}$ be a class of nonnegative functions such that for $g \in \mathcal{A}$ the following conditions hold

1. $g \in \mathcal{C}^{2, \nu}$ is nonnegative and symmetric with respect to zero, i.e $g(-x)=g(x)$. Furthermore, there exists $M>0$ such that

$$
\begin{aligned}
& t^{-\frac{2}{3-\theta}}\left[t^{-\frac{\theta-1}{3-\theta}(2+\nu)} \sup _{\left|x_{1}\right|,\left|x_{2}\right| \leq t^{-\frac{\theta-1}{3-\theta}}}\left(\frac{\left|g_{x x}\left(x_{1}\right)-g_{x x}\left(x_{2}\right)\right|}{\left|x_{1}-x_{2}\right|^{\nu}}\right)\right] \\
& +\sup _{t^{-\frac{\theta-1}{3-\theta}} \leq R \leq 1} R^{\frac{2}{\theta-1}}\left[R^{(2+\nu)} \sup _{R / 2 \leq\left|x_{1}\right|,\left|x_{2}\right| \leq R} \frac{\left|g_{x x}\left(x_{1}\right)-g_{x x}\left(x_{2}\right)\right|}{\left|x_{1}-x_{2}\right|^{\nu}}\right] \leq M .
\end{aligned}
$$

2. There exist $A, B, M>0$ such that

$$
\frac{t^{\frac{2}{3-\theta}}}{M\left(B x^{2} t^{\frac{2(\theta-1)}{3-\theta}}+A\right)^{\frac{1}{\theta-1}}} \leq g(x) \leq \frac{M t^{\frac{2}{3-\theta}}}{\left(B x^{2} t^{\frac{2(\theta-1)}{3-\theta}}+A\right)^{\frac{1}{\theta-1}}} .
$$


3. There exist $A, B, M>0$ such that

$$
\left|g_{x}\right| \leq M \frac{x t^{\frac{2 \theta}{3-\theta}}}{\left(B x^{2} t^{\frac{2(1-\theta)}{3-\theta}}+A\right)^{\frac{\theta}{\theta-1}}} .
$$

4. There exists $\epsilon_{0}>0$ such that

$$
\left|\frac{g_{x}}{g}-\frac{1}{\theta-1} \frac{1}{x}\right| \leq \frac{\epsilon_{0}}{|x|}, \quad\left|\left(\frac{g_{x}}{g}-\frac{1}{\theta-1} \frac{1}{x}\right)_{x}\right| \leq \frac{\epsilon_{0}}{|x|^{2}} .
$$

From now on, and in difference to the previous section, the appearing constants $C=$ $C(\theta, M)$ will depend on $\theta$ and on $M$, as well as the constants denoted by $C_{\delta}, C_{\gamma}$.

Lemma 3.3 The operator $\tilde{A}_{z}(t)$ is self-adjoint with respect to the weighted integral $\frac{d x}{z^{\theta}}$. All eigenvalues are non-positive and the first eigenvalue $\lambda_{0}$ is equal to 0 with corresponding eigenfunction $z^{\theta}$.

Proof. We know that $h_{x}-\theta \frac{z_{x}}{z} h=\left(\frac{h}{z^{\theta}}\right)_{x} z^{\theta}$ for any $h$ and

$$
\int_{I} \tilde{A}_{z}(f) g \frac{d x}{z^{\theta}}=-\int_{I}\left(f_{x}-\theta \frac{z_{x}}{z} f\right)\left(\frac{g}{z^{\theta}}\right)_{x} d x=-\int_{I}\left(\frac{f}{z^{\theta}}\right)_{x}\left(g_{x}-\theta \frac{z_{x}}{z} g\right) d x=\int_{I} f \tilde{A}_{z}(g) \frac{d x}{z^{\theta}} .
$$

It follows from standard arguments that all eigenvalues are non-positive (compare [1]). It is straightforward that $z^{\theta}$ is an eigenvector corresponding to the eigenvalue 0 .

Proposition 3.4 Let $\lambda_{1}$ be the second eigenvalue for the differential operator $\tilde{A}_{z}(t)$. Suppose that $z(x, t)$ satisfies Assumption 3.2. Then there exists an absolute constant $C>0$ independent of $z$ such that

$$
\lambda_{1} \leq-C \quad \text { for all } t
$$

Proof. Suppose that this is not the case. Then there exist a sequence of $t_{m}$, functions $z_{m} \in \mathcal{A}$, and eigenvalues $\lambda_{1, m} \nearrow 0$ for $t_{m} \rightarrow t_{\infty}$ (with $t_{\infty}$ being either finite or infinite), and corresponding eigenfunctions $\phi_{1, m}$ such that

$$
\tilde{A}_{z_{m}}\left(\phi_{1, m}\right)=\left(\phi_{1, m}\right)_{x x}-\theta\left(\frac{\left(z_{m}\right)_{x}}{z_{m}} \phi_{1, m}\right)_{x}=\lambda_{1, m} \phi_{1, m}
$$

Here we assume that the eigenfunction $\phi_{1, m}$ is normalized i.e. $\int\left|\phi_{1, m}\right|^{2} \frac{d x}{z_{m}^{\theta}}=1$.

- If $t_{\infty}<\infty$, then by Assumption 3.2 we have that $\left\|z_{m}\right\|_{\mathcal{C}^{2, \nu}}$ is uniformly bounded, and $z_{m}$ converges to $z_{\infty}$ in $\mathcal{C}^{2}$. Classical regularity theory implies that $\phi_{m} \in \mathcal{C}^{2, \nu}$ and $\left\|\phi_{m}\right\|_{\mathcal{C}^{2, \nu}} \leq C$ for all $m$. Due to Sturm-Liouville theory, the eigenfunctions $\phi_{1, m}$ satisfy 
$\phi_{1, m}(0)=0$ and $\phi_{1, m}(x)>0$ for $x \in(0,1)$. In addition, there exists $\phi_{1, \infty}$ such that $\phi_{1, m} \rightarrow \phi_{1, \infty}$ in $\mathcal{C}^{2}$. Then the limiting equation becomes

$$
\tilde{A}_{z_{\infty}}\left(\phi_{1, \infty}\right)=\left(\phi_{1, \infty}\right)_{x x}-\theta\left(\frac{\left(z_{\infty}\right)_{x}}{z_{\infty}} \phi_{1, \infty}\right)_{x}=0
$$

This equation can be solved explicitly and we obtain

$$
\phi_{1, \infty}(x)=K z_{\infty}^{\theta}(x)+C \int_{0}^{x} \frac{z_{\infty}^{\theta}(x)}{z_{\infty}^{\theta}(\xi)} d \xi .
$$

Since $\phi_{1, \infty}$ is periodic and $z_{\infty}$ is nonnegative, the integral term above must vanish, and thus $\phi_{1, \infty}(x)=K z_{\infty}^{\theta}(x)$. This yields $K=0$, because $\phi_{1, \infty}(0)=0$ and $z_{\infty}(0)>0$. Hence $\phi_{1, \infty}=0$, which contradicts the fact that $\int\left|\phi_{1, \infty}\right|^{2} \frac{d x}{z_{\infty}^{\theta}}=1$.

- The case $t_{\infty}=\infty$. For any $0<\delta<1$ we note that $\left\|z_{m}\right\|_{\mathcal{C}^{2, \nu}(I \backslash[-\delta, \delta])} \leq C_{\delta}$. Let $\delta_{0}>0$ be sufficiently small. Let $\psi(x)=C_{\gamma} x^{\gamma}$ with $\gamma>1$, where $C_{\gamma}$ is a constant satisfying $C_{\gamma} \delta_{0}^{\gamma}=C_{\delta}$ in $\left[0, \delta_{0}\right]$. We show that $\psi \geq z_{m}$ for all $m$. Indeed, for sufficiently small $\epsilon=\epsilon(\gamma)$ we have

$$
\begin{gathered}
\tilde{A}_{z_{m}}(\psi)=\gamma(\gamma-1) x^{\gamma-2}-\frac{\theta}{\theta-1}(\gamma-1) x^{\gamma-2}-\theta\left(\left(\frac{\left(z_{m}\right)_{x}}{z_{m}}-\frac{1}{(\theta-1) x}\right) \psi\right)_{x} \\
=(\gamma-1)\left(\gamma-\frac{\theta}{\theta-1}\right) x^{\gamma-2}-\theta\left(\frac{\left(z_{m}\right)_{x}}{z_{m}}-\frac{1}{(\theta-1) x}\right)_{x} \psi-\theta\left(\frac{\left(z_{m}\right)_{x}}{z_{m}}-\frac{1}{(\theta-1) x}\right) \psi_{x} \\
\leq(\gamma-1)\left(\gamma-\frac{\theta}{\theta-1}\right) x^{\gamma-2}+\theta \frac{\epsilon}{x^{2}} x^{\gamma}+\theta \gamma \frac{\epsilon}{x} x^{\gamma-1} \\
=(\gamma-1)\left(\gamma-\frac{\theta}{\theta-1}+\theta \epsilon \frac{1+\gamma}{\gamma-1}\right) x^{\gamma-2} .
\end{gathered}
$$

So $\tilde{A}_{z_{m}}(\psi) \leq 0$ in $\left[0, \delta_{0}\right]$ for $1<\gamma<\frac{\theta}{\theta-1}$ and so $\psi$ is a super-solution of $\phi_{1, m}$ for all $m$, namely, due to the maximum principle,

$$
\left|\phi_{1, m}(x)\right| \leq C_{\gamma}|x|^{\gamma}, \quad 0 \leq x \leq \delta_{0} .
$$

There exists $\phi_{1, \infty}$ such that $\phi_{1, m} \rightarrow \phi_{1, \infty}$ in $\mathcal{C}^{2}$ over $[\delta, 1]$ for any $0<\delta<1$ and thus the limiting equation becomes

$$
\tilde{A}_{z_{\infty}}\left(\phi_{1, \infty}\right)=\left(\phi_{1, \infty}\right)_{x x}-\theta\left(\frac{\left(z_{\infty}\right)_{x}}{z_{\infty}} \phi_{1, \infty}\right)_{x}=0
$$

As in the previous case, this leads to a contradiction and completes the proof. 
For convenience, we denote $\langle g, h\rangle=\int_{I} g(x) h(x) \frac{d x}{z^{\theta}}$ for functions $g$ and $h$ which are integrable with respect to $d x / z^{\theta}$. We define $v$ in terms of $u:=\frac{z^{\theta}}{\int_{I} z^{\theta} d x}+v$. Note that $\int_{I} v d x=0$ and $\left\langle z^{\theta}, v\right\rangle=0$. Furthermore, $v$ solves

$$
v_{t}=v_{x x}-\theta\left(\frac{z_{x}}{z} v\right)_{x}-\left(\frac{z^{\theta}}{\int_{I} z^{\theta} d x}\right)_{t} .
$$

For simplicity we denote

$$
\mathcal{R}(x, t):=-\left(\frac{z^{\theta}}{\int_{I} z^{\theta} d x}\right)_{t}=-\theta \frac{z^{\theta-1} u}{\int_{I} z^{\theta} d x}+\theta \frac{z^{\theta} \int z^{\theta-1} u d x}{\left(\int_{I} z^{\theta} d x\right)^{2}} .
$$

Now we make an assumption on $v$, which will be recovered in the end.

Assumption 3.5 Suppose that $z(x, t)$ satisfies Assumption 3.2. Further suppose that

$$
|v(x, t)| \leq M \frac{z^{\theta}(x, t)}{\int_{I} z^{\theta}(y, t) d x},
$$

for a suitable constant $M>0$.

Let us first give a useful lemma, which is an adaptation of a result given in [7] and provides one of the main estimates for the result stated thereafter. For the purpose of this paper we use a formulation restated in [4], which is more accessible. The proof of our lemma will be given later in the paper.

Lemma 3.6 Let $1<\theta<3$. Suppose that $\zeta \in C^{1}([0,1])$ with $\zeta(0)=0$, and $z$ satisfy Assumption 3.2 for all $t \geq 1$. Then

$$
\left(\int_{0}^{1} z^{(p-1) \theta}|\zeta|^{p} d x\right)^{\frac{1}{p}} \leq C\left(\int_{0}^{1} z^{\theta}\left|\zeta_{x}\right|^{2} d x\right)^{\frac{1}{2}}, \quad p=\frac{6 \theta-2}{\theta+1} .
$$

With this result we can show a Sobolev inequality with the weighted norm $z^{-\theta}$.

Lemma 3.7 Suppose that $z(x, t)$ satisfies Assumption 3.2, and that $h_{x} \in L^{2}\left(z^{-\theta} d x\right)$ with $\int_{I} h=0$, where $I=[-1,1]$. Then

$$
\left(\int_{I}|h|^{p} \frac{d x}{z^{\theta}}\right)^{\frac{1}{p}} \leq C\left(\int_{I}\left|h_{x}-\theta \frac{z_{x}}{z} h\right|^{2} \frac{d x}{z^{\theta}}\right)^{\frac{1}{2}}, \quad p=\frac{6 \theta-2}{\theta+1} .
$$

Here $C$ is an absolute constant independent of $t, z$, and $\theta$, but depending on $M$.

Proof. We consider the following variational problem:

$$
-\left(h_{x}-\theta \frac{z_{x}}{z} h\right)_{x}=\lambda\left(|h|^{p-2} h-z^{\theta} \frac{\int_{I}|h|^{p-2} h d x}{\int_{I} z^{\theta} d x}\right)
$$


with $\int_{I}|h|^{p} \frac{d x}{z^{\theta}}=1$. Due to Assumption 3.2, $z(x, t)$ is non-singular for every finite $t$, and thus classical theory for semi-linear elliptic boundary value problems with constraints, compare [12] implies that there exists $\lambda(t)>0$ such that

$$
\left(\int_{I}|h|^{p} \frac{d x}{z^{\theta}}\right)^{\frac{1}{p}} \leq \frac{1}{\sqrt{\lambda(t)}}\left(\int_{I}\left|h_{x}-\theta \frac{z_{x}}{z} h\right|^{2} \frac{d x}{z^{\theta}}\right)^{\frac{1}{2}} .
$$

Our goal is to show that there exists $k>0$ such that $\lambda \geq k$ for all $t \geq t_{0}$.

Suppose that this not the case. Then there exist $t_{n}, z_{n}, h_{n}$ with $\int_{I}\left|h_{n}\right|^{p} \frac{d x}{z_{n}^{\theta}}=1$ and $\lambda_{n}\left(t_{n}\right) \searrow 0$ for $t_{n} \rightarrow \infty$, possibly after choosing a suitable subsequence, such that

$$
\lambda_{n}=\int_{I}\left|\left(h_{n}\right)_{x}-\theta \frac{\left(z_{n}\right)_{x}}{z_{n}} h_{n}\right|^{2} \frac{d x}{z_{n}^{\theta}} .
$$

We introduce a new function $\varphi_{n}:=\frac{h_{n}}{z_{n}^{\theta}}$. Then, since we want to minimize the constant $C$ in (18), the given problem can be rewritten as an eigenvalue problem

$$
-\left(z_{n}^{\theta}\left(\varphi_{n}\right)_{x}\right)_{x}=\lambda_{n}\left[z_{n}^{(p-1) \theta}\left|\varphi_{n}\right|^{p-2} \varphi_{n}-\frac{\int_{I} z_{n}^{(p-1) \theta}\left|\varphi_{n}\right|^{p-2} \varphi_{n} d x}{\int_{I} z_{n}^{\theta} d x} z_{n}^{\theta}\right],
$$

because

$$
\int_{I}\left|h_{n}\right|^{p} \frac{d x}{z_{n}^{\theta}}=\int_{I}\left|\varphi_{n}\right|^{p} z_{n}^{(p-1) \theta} d x .
$$

Additionally, the normalization and orthogonality condition have to be fulfilled, namely

$$
\int_{I} z_{n}^{(p-1) \theta}\left|\varphi_{n}\right|^{p} d x=1, \quad \int_{I} z_{n}^{\theta} \varphi_{n} d x=0, \quad \text { and } \quad \lambda_{n}=\int_{I} z_{n}^{\theta}\left|\left(\varphi_{n}\right)_{x}\right|^{2} d x .
$$

Now expressing $\varphi_{n}=\varphi_{n}(0)+\psi_{n}$ we can estimate (19) by

$$
\int_{I}\left|\varphi_{n}\right|^{p} z_{n}^{(p-1) \theta} d x \leq \int_{I}\left|\varphi_{n}(0, t)\right|^{p} z_{n}^{(p-1) \theta} d x+\int_{I}\left|\psi_{n}(x, t)\right|^{p} z_{n}^{(p-1) \theta} d x .
$$

To control the first term on the right hand side of $(20)$, we estimate $\left|\varphi_{n}(0, t)\right|$. First, by Hölder's inequality we obtain

$$
\begin{array}{r}
\left|\varphi_{n}(x, t)-\varphi_{n}(0, t)\right|=\left|\int_{0}^{x}\left(\varphi_{n}\right)_{x}(\xi, t) d \xi\right| \leq\left(\int_{0}^{x}\left|\left(\varphi_{n}\right)_{x}\right|^{2} z_{n}^{\theta} d \xi\right)^{\frac{1}{2}}\left(\left|\int_{0}^{x} \frac{d \xi}{z_{n}^{\theta}}\right|\right)^{\frac{1}{2}} \\
\leq \sqrt{\lambda_{n}}\left(\left|\int_{0}^{x} \frac{d \xi}{z_{n}^{\theta}}\right|\right)^{\frac{1}{2}}
\end{array}
$$

Therefore, we have

$$
\left|\varphi_{n}(x, t)\right| \leq\left|\varphi_{n}(0, t)\right|+\sqrt{\lambda_{n}}\left(\left|\int_{0}^{x} \frac{d \xi}{z_{n}^{\theta}(\xi, t)}\right|\right)^{\frac{1}{2}} .
$$


Due to (21), it is direct that $\left|\psi_{n}\right| \leq \sqrt{\lambda_{n}}\left(\left|\int_{0}^{x} \frac{d \xi}{z_{n}^{\theta}(\xi, t)}\right|\right)^{\frac{1}{2}}$. Using $0=\int_{I} z_{n}^{\theta} \varphi_{n} d x=$ $\varphi_{n}(0, t) \int_{I} z_{n}^{\theta} d x+\int_{I} z_{n}^{\theta} \psi_{n} d x$, we obtain due to $(22)$

$$
\left|\varphi_{n}(0, t)\right|=\left|\frac{\int_{I} z_{n}^{\theta} \psi_{n} d x}{\int_{I} z_{n}^{\theta} d x}\right| \leq \frac{\sqrt{\lambda_{n}} \int_{I} z_{n}^{\theta}\left(\left|\int_{0}^{x} \frac{d \xi}{z_{n}^{\theta}}\right|\right)^{\frac{1}{2}} d x}{t^{\frac{\theta+1}{3-\theta}}} \leq C \sqrt{\lambda_{n}} t^{-\frac{\theta+1}{3-\theta}}
$$

Here we used that $\int_{I} z_{n}^{\theta}\left(\int_{I} \frac{d \xi}{z_{n}^{\theta}}\right)^{\frac{1}{2}} d x \leq C$. Due to Assumption 3.2, 2., we compute

$$
\int_{I} z_{n}^{(p-1) \theta}\left|\varphi_{n}(0)\right|^{p} d x \leq C \lambda_{n}^{\frac{p}{2}} t^{-\frac{(\theta+1) p}{3-\theta}} \int_{I} z_{n}^{(p-1) \theta} d x \leq C \lambda_{n}^{\frac{p}{2}} t^{-\frac{3 \theta-1}{1+\theta}}
$$

where we used $\int_{I}\left(y^{2}+a\right)^{-\frac{\theta(p-1)}{\theta-1}} d y<\infty$. Now we estimate the second term in (20). This is done by Lemma 3.6. For $p>2$ we obtain

$$
\begin{gathered}
\left(\int_{I} z_{n}^{(p-1) \theta}\left|\varphi_{n}\right|^{p} d x\right)^{\frac{1}{p}} \leq\left(\int_{I} z_{n}^{(p-1) \theta}\left|\varphi_{n}(0, t)\right|^{p} d x\right)^{\frac{1}{p}}+\left(\int_{I} z_{n}^{(p-1) \theta}\left|\psi_{n}\right|^{p} d x\right)^{\frac{1}{p}} \\
\leq C \lambda_{n}^{\frac{p}{2}} t^{-\frac{3 \theta-1}{1+\theta}}+C\left(\int_{I} z_{n}^{\theta}\left|\left(\psi_{n}\right)_{x}\right|^{2} d x\right)^{\frac{1}{2}} \leq C \lambda_{n} \rightarrow 0 \quad \text { for } n \rightarrow \infty
\end{gathered}
$$

This is a contradiction to our hypothesis and thus completes the proof of Lemma 3.7.

Now we give the proof of Lemma 3.6. If $z$ would behave like a power law, we could have mainly used the estimate given in [4] to obtain our result. But unfortunately this is not the case everywhere, so that we have to introduce boundary layer estimates.

\section{Proof of Lemma 3.6}

For convenience, we denote $\alpha=\frac{\theta-1}{3-\theta}$. First the contributions where $z$ is large are analyzed. For this, as can be seen from Assumption 3.2, we have to look at a specific domain of integration. So we show that for a smooth function $\zeta$ with $\zeta(0)=0$

$$
\left(\int_{0}^{2 t^{-\alpha}} z^{(p-1) \theta}|\zeta|^{p} d x\right)^{\frac{1}{p}} \leq C\left(\int_{0}^{2 t^{-\alpha}} z^{\theta}\left|\zeta_{x}\right|^{2} d x\right)^{\frac{1}{2}}
$$

Indeed, due to Assumption 3.2 and with the change of variables $y=t^{\alpha} x$ and $\tilde{\zeta}(y)=$ $\zeta\left(t^{-\alpha} y\right)$, we have

$$
\left(\int_{0}^{2 t^{-\alpha}} z^{(p-1) \theta}|\zeta|^{p} d x\right)^{\frac{1}{p}} \leq\left(\int_{0}^{2 t^{-\alpha}} \frac{t^{\frac{2(p-1) \theta}{3-\theta}}}{\left(x^{2} t^{2 \alpha}+a\right)^{\frac{(p-1) \theta}{\theta-1}}}|\zeta|^{p} d x\right)^{\frac{1}{p}}
$$




$$
\begin{gathered}
=t^{\frac{2(p-1) \theta}{3-\theta}-\frac{\alpha}{p}}\left(\int_{0}^{2} \frac{1}{\left(y^{2}+a\right)^{\frac{(p-1) \theta}{\theta-1}}}|\tilde{\zeta}|^{p} d y\right)^{\frac{1}{p}} \leq C t^{\frac{2(p-1) \theta}{3-\theta}-\frac{\alpha}{p}}\left(\int_{0}^{2} \frac{1}{\left(y^{2}+a\right)^{\frac{p \theta}{\theta-1}}}\left|\tilde{\zeta}_{x}\right|^{2} d y\right)^{\frac{1}{2}} \\
\leq C t^{\frac{2(p-1) \theta}{3-\theta}-\frac{\alpha}{p}-\frac{\theta}{3-\theta}-\frac{\alpha}{2}}\left(\int_{0}^{2 t^{-\alpha}} z^{\theta}\left|\zeta_{x}\right|^{2} d x\right)^{\frac{1}{2}} \leq C\left(\int_{0}^{2 t^{-\alpha}} z^{\theta}\left|\zeta_{x}\right|^{2} d x\right)^{\frac{1}{2}}
\end{gathered}
$$

since $\frac{2(p-1) \theta}{3-\theta}-\frac{\alpha}{p}-\frac{\theta}{3-\theta}-\frac{\alpha}{2}=0$. Next we do a further splitting

$$
\zeta(x, t)=\eta\left(x t^{\alpha}\right) \zeta(x, t)+\left(1-\eta\left(x t^{\alpha}\right)\right) \zeta(x, t):=\tilde{\zeta}(x, t)+\hat{\zeta}(x, t),
$$

where $\eta$ is a standard cut-off function such that $\eta(y)=1$ for $y \leq 1$ and $\eta=0$ if $y \geq 2$. Then, since $\tilde{\zeta}$ is supported in $\left[0,2 t^{-\alpha}\right)$ and $\tilde{\zeta}(0)=0$, using (23), we get

$$
\left(\int_{0}^{1} z^{(p-1) \theta}|\tilde{\zeta}|^{p} d x\right)^{\frac{1}{p}} \leq C\left(\int_{0}^{1} z^{\theta}\left|\tilde{\zeta}_{x}\right|^{2} d x\right)^{\frac{1}{2}}
$$

Because $\tilde{\zeta}_{x}=\eta\left(x t^{\alpha}\right) \zeta_{x}+t^{\alpha} \eta^{\prime}\left(x t^{\alpha}\right) \zeta$, we obtain

$$
\left(\int_{0}^{1} z^{(p-1) \theta}|\tilde{\zeta}|^{p} d x\right)^{\frac{1}{p}} \leq C\left(\int_{0}^{1} z^{\theta}\left|\zeta_{x}\right|^{2} d x\right)^{\frac{1}{2}}+C\left(\int_{0}^{1} z^{\theta} t^{2 \alpha}\left|\eta^{\prime} \zeta\right|^{2} d x\right)^{\frac{1}{2}}
$$

We now give an estimate for the second term on the right hand side of (24). Noting that $\eta^{\prime}$ is supported in $\left(t^{-\alpha}, 2 t^{-\alpha}\right)$, we compute

$$
\begin{gathered}
\left(\int_{0}^{1} z^{\theta} t^{2 \alpha}\left|\eta^{\prime} \zeta\right|^{2} d x\right)^{\frac{1}{2}} \leq\left(\int_{t^{-\alpha}}^{2 t^{-\alpha}} \frac{t^{\frac{2 \theta}{3-\theta}}}{\left(x^{2} t^{2 \alpha}+a\right)^{\frac{\theta}{\theta-1}}} t^{2 \alpha}|\zeta|^{2} d x\right)^{\frac{1}{2}} \\
=t^{\frac{2 \theta}{3-\theta}+\frac{\beta}{2}}\left(\int_{1}^{2} \frac{1}{\left(y^{2}+a\right)^{\frac{\theta}{\theta-1}}}|\zeta|^{2} d y\right)^{\frac{1}{2}} \leq t^{\frac{2 \theta}{3-\theta}+\frac{\beta}{2}}\left(\int_{0}^{2} \frac{1}{\left(y^{2}+a\right)^{\frac{\theta}{\theta-1}}}|\zeta|^{2} d y\right)^{\frac{1}{2}} \\
\leq C t^{\frac{2 \theta}{3-\theta}+\frac{\beta}{2}}\left(\int_{0}^{2} \frac{1}{\left(y^{2}+a\right)^{\frac{\theta}{\theta-1}}}\left|\zeta_{y}\right|^{2} d y\right)^{\frac{1}{2}} \leq C\left(\int_{0}^{2 t^{-\alpha}} z^{\theta}\left|\zeta_{x}\right|^{2} d x\right)^{\frac{1}{2}},
\end{gathered}
$$

where we used $\psi_{n}(0)=0$.

Combining (24) and (25), we obtain

$$
\left(\int_{I} z^{(p-1) \theta}|\tilde{\zeta}|^{p} d x\right)^{\frac{1}{p}} \leq C\left(\int_{I} z^{\theta}\left|\zeta_{x}\right|^{2} d x\right)^{\frac{1}{2}} .
$$


It remains to show that

$$
\left(\int_{I} z^{(p-1) \theta}|\hat{\zeta}|^{p} d x\right)^{\frac{1}{p}} \leq C\left(\int_{I} z^{\theta}\left|\zeta_{x}\right|^{2} d x\right)^{\frac{1}{2}} .
$$

Keeping in mind that $\hat{\zeta}$ vanishes in $\left[0, t^{-\alpha}\right]$, we note that $z$ is comparable to $|x|^{-\frac{2}{\theta-1}}$. It is direct that $z \leq C|x|^{\frac{2}{\theta-1}}$. On the other hand, since $x>t^{-\alpha}$, we have $|x|^{-\frac{2}{\theta-1}} \leq$ $C\left(x^{2}+t^{-\alpha}\right)^{-\frac{1}{\theta-1}} \leq C z$. Therefore,

$$
\begin{aligned}
& \left(\int_{I} z^{(p-1) \theta}|\hat{\zeta}|^{p} d x\right)^{\frac{1}{p}} \leq C\left(\int_{I} x^{-\frac{2(p-1) \theta}{\theta-1}}|\hat{\zeta}|^{p} d x\right)^{\frac{1}{p}} \\
& \leq C\left(\int_{I} x^{-\frac{2 \theta}{\theta-1}}\left|\hat{\zeta}_{x}\right|^{2} d x\right)^{\frac{1}{2}} \leq C\left(\int_{I} z^{\theta}\left|\hat{\zeta}_{x}\right|^{2} d x\right)^{\frac{1}{2}},
\end{aligned}
$$

where we used a known Sobolev inequality with weight (see e.g. [[7], Theorem 1 and corollaries in 2]).

For $\tilde{\zeta}$, we note that $\hat{\zeta}_{x}=\left(1-\eta\left(x t^{\alpha}\right)\right) \zeta_{x}-t^{\alpha} \eta^{\prime}\left(x t^{\alpha}\right) \zeta$.

Following a similar procedure as for the estimate (25), we can show without giving further details, that

$$
\left(\int_{I} z^{\theta}\left|\hat{\zeta}_{x}\right|^{2} d x\right)^{\frac{1}{2}} \leq C\left(\int_{I} z^{\theta}\left|\zeta_{x}\right|^{2} d x\right)^{\frac{1}{2}}
$$

Summarizing the above estimates, we obtain

$$
\left(\int_{I} z^{(p-1) \theta}|\hat{\zeta}|^{p} d x\right)^{\frac{1}{p}} \leq C\left(\int_{I} z^{\theta}\left|\zeta_{x}\right|^{2} d x\right)^{\frac{1}{2}} .
$$

Then estimates (26) and (27) lead to

$$
\begin{aligned}
\left(\int_{0}^{1} z^{(p-1) \theta}|\zeta|^{p} d x\right)^{\frac{1}{p}} & \leq\left(\int_{0}^{1} z^{(p-1) \theta}|\tilde{\zeta}|^{p} d x\right)^{\frac{1}{p}}+\left(\int_{0}^{1} z^{(p-1) \theta}|\hat{\zeta}|^{p} d x\right)^{\frac{1}{p}} \\
& \leq C\left(\int_{0}^{1} z^{\theta}\left|\zeta_{x}\right|^{2} d x\right)^{\frac{1}{2}} .
\end{aligned}
$$

This completes the proof of our lemma on the extension of the result given in [4].

Lemma 3.8 Suppose that $z(x, t)$ and $v(x, t)$ satisfy the Assumption 3.2 and the Assumption 3.5, respectively. Then

$$
\langle v, v\rangle \leq \frac{C}{t^{\frac{\theta+5}{3-\theta}}} \quad \text { and } \quad|v(x, t)| \leq \frac{C}{t^{\frac{\theta+5}{2(3-\theta)}}} \quad \text { for } \quad|x|>\delta .
$$


Proof. First we see, that

$$
\int_{I} v v_{t} \frac{d x}{z^{\theta}}=\int_{I}\left(v_{x x}-\theta\left(\frac{z_{x}}{z} v\right)_{x}\right) v \frac{d x}{z^{\theta}}+\int_{I} \mathcal{R}(x, t) v \frac{d x}{z^{\theta}}=<v, \tilde{A}_{z}(v)>+<\mathcal{R}, v>.
$$

Now we split the first term on the right hand side into half and since $\left\langle z^{\theta}, v\right\rangle=0$, due to Proposition 3.4, we have $\frac{1}{2}<v, \tilde{A}_{z}(v)>\leq-C<v, v>$ and thus obtain

$$
\frac{d}{d t}<v, v>+\frac{C}{2}<v, v>+\frac{1}{2}<v_{x}-\theta \frac{z_{x}}{z} v, v_{x}-\theta \frac{z_{x}}{z} v>\leq|<\mathcal{R}, v>|+\frac{C}{t}<v, v>.
$$

Using Hölder's inequality and (18), we have

$$
\begin{aligned}
& \frac{d}{d t}\|v\|_{L^{2}\left(z^{-\theta} d x\right)}^{2}+C\|v\|_{L^{2}\left(z^{-\theta} d x\right)}^{2}+C\left\|v_{x}-\theta \frac{z_{x}}{z} v\right\|_{L^{2}\left(z^{-\theta} d x\right)}^{2} \leq\|\mathcal{R}\|_{L^{p^{\prime}\left(z^{-\theta} d x\right)}}\|v\|_{L^{p}\left(z^{-\theta} d x\right)} \\
& \leq C_{\epsilon}\|\mathcal{R}\|_{L^{p^{\prime}\left(z^{-\theta} d x\right)}}^{2}+\epsilon\|v\|_{L^{p}\left(z^{-\theta} d x\right)}^{2} \leq C_{\epsilon}\|\mathcal{R}\|_{L^{p^{\prime}\left(z^{-\theta} d x\right)}}^{2}+C \cdot \epsilon\left\|v_{x}-\theta \frac{z_{x}}{z} v\right\|_{L^{2}\left(z^{-\theta} d x\right)}^{2},
\end{aligned}
$$

where $p$ is given as in (18) and $p^{\prime}=(6 \theta-2) /(5 \theta-3)$ is its Hölder conjugate. Summing up, we obtain

$$
\frac{d}{d t}\|v\|_{L^{2}\left(z^{-\theta} d x\right)}^{2}+C\|v\|_{L^{2}\left(z^{-\theta} d x\right)}^{2}+C\left\|v_{x}-\theta \frac{z_{x}}{z} v\right\|_{L^{2}\left(z^{-\theta} d x\right)}^{2} \leq C_{\epsilon}\|\mathcal{R}\|_{L^{p^{\prime}\left(z^{-\theta} d x\right)}}^{2} .
$$

Due to Assumption 3.2, we compute

$$
\begin{aligned}
\|\mathcal{R}\|_{L^{p^{\prime}\left(z^{-\theta} d x\right)}}^{2} & =\left(\int\left|\frac{z^{\theta-1} u}{\int z^{\theta} d x}\right|^{p^{\prime}} \frac{d x}{z^{\theta}}\right)^{\frac{2}{p^{\prime}}} \leq \frac{C}{\left(\int z^{\theta} d x\right)^{4}}\left(\int z^{(2 \theta-1) p^{\prime}-\theta} d x\right)^{\frac{2}{p^{\prime}}} \\
& \leq C t^{-\frac{4(\theta+1)}{3-\theta}} t^{\frac{4(2 \theta-1) p^{\prime}-\theta(3-\theta)-(\theta-1)}{p^{\prime}(3-\theta)}} \leq C t^{-\frac{\theta+5}{3-\theta}} .
\end{aligned}
$$

Therefore, $\langle v, v\rangle \leq C t^{-\frac{\theta+5}{3-\theta}}$ and so $|v(x, t)| \leq C t^{-\frac{\theta+5}{2(3-\theta)}}$ for $|x|>\delta$. This completes the proof.

\subsection{Estimates for the solution near $x=0$}

We introduce an internal variable in the following way:

$$
\xi=t^{\frac{\theta-1}{3-\theta}} x, \quad v(x, t)=t^{\frac{\theta-1}{3-\theta}} G(\xi, t), \quad z(x, t)=t^{\frac{2}{3-\theta}} Z(\xi, t),
$$

and let $\alpha=(\theta-1) /(3-\theta)$ and $\gamma=2 /(3-\theta)$. Due to Lemma 3.8, we have

$$
|G(\xi, t)| \leq C t^{-\frac{\theta+5}{2(3-\theta)}-\frac{\theta-1}{3-\theta}}=C t^{\frac{-\theta-5-2 \theta+2}{2(3-\theta)}}=C t^{-\frac{3(\theta+1)}{2(3-\theta)}} \quad \text { for }|\xi| \geq t^{\alpha} \delta \quad \text { for any } \delta>0 .
$$


If $z$ satisfies Assumption 3.2, then $Z(\xi, t) \approx\left(\xi^{2}+a\right)^{-\frac{1}{\theta-1}}$. Furthermore, under Assumption 3.5 , one can easily see that

$$
|G(\xi, t)| \leq M \frac{Z^{\theta}}{\Gamma(t)}, \quad \text { with } \Gamma(t)=\int_{-t^{\alpha}}^{t^{\alpha}} Z^{\theta}(\xi, t) d \xi .
$$

Since $\Gamma(t) \leq C$ for all $t>0$, we obtain $|G(\xi, t)| \leq C M Z^{\theta}$. Recalling (17), in terms of the new variables, simple computations show that

$$
\mathcal{R}(\xi, t)=\theta t^{\alpha-1}\left(\frac{Z^{2 \theta-1}}{\Gamma^{2}(t)}+\frac{Z^{\theta-1} G}{\Gamma^{2}(t)}+\frac{Z^{\theta} \Lambda(t)}{\Gamma^{3}(t)}+\frac{Z^{\theta} \Upsilon(t)}{\Gamma^{2}(t)}\right),
$$

where

$$
\Lambda(t)=\int_{-t^{\alpha}}^{t^{\alpha}} Z^{2 \theta-1}(\xi, t) d \xi, \quad \Upsilon(t)=\int_{-t^{\alpha}}^{t^{\alpha}} Z^{\theta-1}(\xi, t) G(\xi, t) d \xi
$$

It is direct that $\Gamma(t), \Lambda(t)$, and $\Upsilon(t)$ are uniformly bounded for any $t$ as long as $z$ and $v$ satisfy Assumptions 3.2 and 3.5, respectively. For convenience, denote $\mathcal{R}=t^{\alpha-1} \mathcal{R}_{1}+$ $\theta t^{\alpha-1} Z^{\theta-1} \Gamma^{-2}(t) G$, where

$$
\mathcal{R}_{1}(\xi, t)=\theta\left(\frac{Z^{2 \theta-1}}{\Gamma^{2}(t)}+\frac{Z^{\theta} \Lambda(t)}{\Gamma^{3}(t)}+\frac{Z^{\theta} \Upsilon(t)}{\Gamma^{2}(t)}\right) .
$$

By change of variable, due to (16), $G$ solves

$$
\frac{\theta-1}{3-\theta} t^{\frac{\theta-1}{3-\theta}-1}\left(G+\xi \frac{\partial G}{\partial \xi}\right)+t^{\frac{\theta-1}{3-\theta}} \frac{\partial G}{\partial t}=t^{\frac{3(\theta-1)}{3-\theta}}\left[G_{\xi \xi}-\theta\left(G \frac{Z_{\xi}}{Z}\right)_{\xi}\right]+\mathcal{R}(\xi, t) .
$$

Simplifying (29), we have

$$
G_{\xi \xi}-\theta\left(G \frac{Z_{\xi}}{Z}\right)_{\xi}=\alpha t^{-2 \alpha-1}\left(G+\xi \frac{\partial G}{\partial \xi}\right)+t^{-2 \alpha} \frac{\partial G}{\partial t}-\theta t^{-2 \alpha-1} \frac{Z^{\theta-1}}{\Gamma^{2}(t)} G+t^{-2 \alpha-1} \mathcal{R}_{1} .
$$

The next Lemma shows the asymptotic behavior of $u$ under Assumption 3.2.

Lemma 3.9 If $z(x, t)$ satisfies Assumption 3.2, then

$$
\left|u(x, t)-\frac{z^{\theta}}{\int_{I} z^{\theta} d x}\right| \leq \varepsilon(t) \frac{z^{\theta}}{\int_{I} z^{\theta} d x},
$$

where $\varepsilon(t) \leq C t^{-\beta}$ for some $\beta>0$.

The proof of Lemma 3.9 relies on

Lemma 3.10 Suppose that $z(x, t)$ and $v(x, t)$ satisfy Assumption 3.2 and Assumption 3.5, respectively. Let $G$ be defined as in (28) so that $G$ solves the equation (29). Then there exists a super solution for $G$ in the set $\xi \leq \delta t^{(\theta-1) /(3-\theta)}$ with a sufficiently small $\delta>0$. 
Proof. First, we look for a super-solution of the form

$$
\mathcal{G}_{1}(\xi, t):=\hat{S}(\xi, t)+\hat{U}(\xi, t)=t^{-(2 \alpha+1)} S(\xi, t)+t^{-2(2 \alpha+1)} U(\xi, t),
$$

where

$$
S(\xi, t)=Z^{\theta}(\xi, t) \int_{0}^{\xi} Z^{-\theta}(y, t) \int_{y}^{\infty} \mathcal{R}_{1}(\eta, t) d \eta d y
$$

and $\hat{U}$ solves

$$
U_{\xi \xi}-\theta\left(U \frac{Z_{\xi}}{Z}\right)_{\xi}=\frac{K_{1}}{\left(\xi^{2}+a\right)^{\frac{1}{\theta-1}}}
$$

Here $K_{1}$ is a constant, which will be specified later. Since $\mathcal{R} \approx Z^{\theta}$, up to multiplicative constants depending on $M$, one can check that $S_{\xi \xi}-\theta\left(\frac{Z_{\xi}}{Z} S\right)_{\xi} \approx \mathcal{R}_{1}$ and

$$
|S|+\left|\xi S_{\xi}\right| \approx \frac{1}{\left(\xi^{2}+a\right)^{\frac{1}{\theta-1}}}, \quad\left|S_{t}\right| \approx \frac{1}{t\left(\xi^{2}+a\right)^{\frac{1}{\theta-1}}}
$$

again, all up to multiplicative constants depending on $M$. Similarly, we can show

$$
|U|+\left|\xi U_{\xi}\right| \approx \frac{1}{\left(\xi^{2}+a\right)^{\frac{2-\theta}{\theta-1}}}, \quad\left|U_{t}\right| \approx \frac{1}{t\left(\xi^{2}+a\right)^{\frac{2-\theta}{\theta-1}}} .
$$

Now we define a differential operator $\mathcal{H}_{Z}$ as follows:

$$
\mathcal{H}_{Z}(f)=-t^{-2 \alpha} \frac{\partial f}{\partial t}+f_{\xi \xi}-\theta\left(\frac{Z_{\xi}}{Z} f\right)_{\xi}-\alpha t^{-2 \alpha-1}\left(f+\xi \frac{\partial f}{\partial \xi}\right)+\theta t^{-2 \alpha-1} \frac{Z^{\theta-1}}{\Gamma^{2}(t)} f .
$$

And compute

$$
\begin{gathered}
\mathcal{H}_{Z}\left(\mathcal{G}_{1}\right)=-t^{-2 \alpha} \frac{\partial \mathcal{G}_{1}}{\partial t}+t^{-2 \alpha-1)} \mathcal{R}_{1}+t^{-2(2 \alpha+1)} \frac{K_{1}}{\left(\xi^{2}+a\right)^{\frac{1}{\theta-1}}} \\
-\alpha t^{-2 \alpha-1}\left(\mathcal{G}_{1}+\xi \frac{\partial \mathcal{G}_{1}}{\partial \xi}\right)+\theta t^{-2 \alpha-1} \frac{Z^{\theta-1}}{\Gamma^{2}(t)} \mathcal{G}_{1} \\
=t^{-2(2 \alpha+1)}\left(\frac{K_{1}}{\left(\xi^{2}+a\right)^{\frac{1}{\theta-1}}}-\alpha\left(S+\xi S_{\xi}\right)+\theta Z^{\theta-1} S-t^{2 \alpha+2} \frac{\partial}{\partial t}\left(t^{-(2 \alpha+1)} S\right)\right) \\
+t^{-3(2 \alpha+1)}\left(\theta Z^{\theta-1} U-\alpha\left(U+\xi U_{\xi}\right)-t^{2 \alpha+2} \frac{\partial}{\partial t}\left(t^{-2(2 \alpha+1)} U\right)\right)+t^{-2 \alpha-1} \mathcal{R}_{1} . \\
\approx t^{-2(2 \alpha+1)}\left(K_{1}+1\right)\left(\xi^{2}+a\right)^{-\frac{1}{\theta-1}}+t^{-3(2 \alpha+1)}\left(\xi^{2}+a\right)^{-\frac{2-\theta}{\theta-1}}+t^{-2 \alpha-1} \mathcal{R}_{1},
\end{gathered}
$$

where we used (30) and (31) and where $C_{\alpha, \theta}$ is a constant depending on $\alpha$ and $\theta$. 
Next we are looking for a super-solution of (29), which is of the form

$$
\bar{G}(\xi, t)=\gamma(t) Z^{\theta}(\xi, t)+t^{-2 \alpha-1} \gamma(t) \psi(\xi, t)+e^{-\mu(t-\bar{t})} Q(\xi, t),
$$

where $\bar{t}$ in the last term is fixed and

$$
\gamma(t)=C_{\delta} t^{-\frac{1}{2}}, \quad \psi(\xi, t)=K_{2} Z^{\theta}(\xi, t) \int_{0}^{\xi} Z^{-\theta}(y, t) \int_{y}^{\infty} Z^{\theta}(\nu, t) d \nu d y
$$

with constants $K_{2}$ and $\mu$, which will be specified later, and $C_{\delta}$ with $C_{\delta}>2 C$, where $C$ is the absolute constant appearing in Lemma 3.8. For convenience, denote

$$
\mathcal{G}_{2}:=\gamma(t) Z^{\theta}(\xi, t)+t^{-2 \alpha-1} \gamma(t) \psi(\xi, t), \quad \mathcal{G}_{3}:=e^{-\mu(t-\bar{t})} Q(\xi, t) .
$$

We have

$$
\begin{gathered}
\psi_{\xi \xi}-\theta\left(\frac{Z_{\xi}}{Z} \psi\right)_{\xi}=\frac{K_{2}}{\left(\xi^{2}+a\right)^{\frac{\theta}{\theta-1}}}, \\
|\psi|+\left|\xi \psi_{\xi}\right| \approx \frac{1}{\left(\xi^{2}+a\right)^{\frac{1}{\theta-1}}}, \quad\left|\psi_{t}\right| \approx \frac{1}{t\left(\xi^{2}+a\right)^{\frac{1}{\theta-1}}} .
\end{gathered}
$$

With (33) and (34), we can show that

$$
\begin{aligned}
\mathcal{H}_{Z}\left(\mathcal{G}_{2}\right)= & -t^{-2 \alpha} \frac{\partial \mathcal{G}_{2}}{\partial t}+t^{-2 \alpha-1} \gamma(t) \frac{K_{2}}{\left(\xi^{2}+a\right)^{\frac{\theta}{\theta-1}}}-\alpha t^{-2 \alpha-1}\left(\mathcal{G}_{2}+\xi \frac{\partial \mathcal{G}_{2}}{\partial \xi}\right)+t^{-2 \alpha-1} \theta Z^{\theta-1} \mathcal{G}_{2} \\
& \approx t^{-2 \alpha-1} \gamma(t) \frac{1+K_{2}}{\left(\xi^{2}+a\right)^{\frac{\theta}{\theta-1}}}+t^{-4 \alpha-2} \gamma(t)\left(\frac{1}{\left(\xi^{2}+a\right)^{\frac{\theta}{\theta-1}}}+\frac{1}{\left(\xi^{2}+a\right)^{\frac{1}{\theta-1}}}\right) .
\end{aligned}
$$

Finally, choose $\mathcal{G}_{3}=e^{-\mu(t-\bar{t})} Q(\xi, t)$ where $Q$ satisfies

$$
-t^{-2 \alpha} Q_{t}+Q_{\xi \xi}-\theta\left(Q \frac{Z_{\xi}}{Z}\right)_{\xi} \leq \alpha t^{-2 \alpha-1}\left(Q+\xi \frac{\partial Q}{\partial \xi}\right)-\theta t^{-2 \alpha-1} \frac{Z^{\theta-1}}{\Gamma^{2}(t)} Q-\mu t^{-2 \alpha} Q
$$

as well as $Q(\xi, t)>0$ on the boundary $|\xi|=\delta t^{\frac{\theta-1}{3-\theta}}$. We obtain a solution satisfying (36) in a perturbative manner. To do this we take $Q_{0}(\xi, t)=a(Z(\xi, t))^{\theta}$, where $a$ is a constant of order one to be determined. Then $Q_{0}$ solves

$$
Q_{0, \xi \xi}-\theta\left(Q_{0} \frac{Z_{\xi}}{Z}\right)_{\xi}=0
$$

We look for solutions of (36) of the form

$$
Q(\xi, t)=Q_{0}(\xi, t)+Q_{1}(\xi, t),
$$


where $Q_{0}$ is given in (37) and $Q_{1}$ satisfies

$$
\begin{gathered}
Q_{1, \xi \xi}-\theta\left(Q_{1} \frac{Z_{\xi}}{Z}\right)_{\xi} \leq-2\left(\alpha t^{-2 \alpha-1} Q_{0}+\alpha t^{-2 \alpha-1} \xi\left|\frac{\partial Q_{0}}{\partial \xi}\right|\right. \\
\left.+\theta t^{-2 \alpha-1} \frac{Z^{\theta-1}}{\Gamma^{2}(t)} Q_{0}+\mu t^{-2 \alpha} Q_{0}+t^{-2 \alpha}\left|Q_{0, t}\right|\right)
\end{gathered}
$$

with $Q_{1}(0, t)=0$. Now it remains show that $Q=Q_{0}+Q_{1}$ satisfies (36). Suppose that $\delta$ is sufficiently small. Assume that $Q_{1}$ satisfies

$$
\left|Q_{1}\right| \leq Q_{0}, \quad\left|\frac{\partial Q_{1}}{\partial \xi}\right| \leq\left|\frac{\partial Q_{0}}{\partial \xi}\right|, \quad\left|\frac{\partial Q_{1}}{\partial t}\right| \leq\left|\frac{\partial Q_{0}}{\partial t}\right|
$$

in the set $|\xi| \leq \delta t^{\frac{\theta-1}{3-\theta}}$. We will check this condition "a posteriori". First we prove (36). We compute the following quantity

$$
J \equiv-t^{-2 \alpha} Q_{t}+Q_{\xi \xi}-\theta\left(Q \frac{Z_{\xi}}{Z}\right)_{\xi}-\alpha t^{-2 \alpha-1}\left(Q+\frac{\partial Q}{\partial \xi}\right)+\theta t^{-2 \alpha-1} \frac{Z^{\theta-1}}{\Gamma^{2}(t)} Q+\mu t^{-2 \alpha} Q .
$$

Using $Q=Q_{0}+Q_{1}$ as well as (37), we obtain

$$
\begin{gathered}
J=Q_{1, \xi \xi}-\theta\left(Q_{1} \frac{Z_{\xi}}{Z}\right)_{\xi}-t^{-2 \alpha} Q_{0, t}-t^{-2 \alpha} Q_{1, t}-\alpha t^{-2 \alpha-1}\left(Q_{0}+\frac{\partial Q_{0}}{\partial \xi}\right) \\
-\alpha t^{-2 \alpha-1}\left(Q_{1}+\frac{\partial Q_{1}}{\partial \xi}\right)+\theta t^{-2 \alpha-1} \frac{Z^{\theta-1}}{\Gamma^{2}(t)} Q_{0}+\theta t^{-2 \alpha-1} \frac{Z^{\theta-1}}{\Gamma^{2}(t)} Q_{1}+\mu t^{-2 \alpha} Q_{0}+\mu t^{-2 \alpha} Q_{1} .
\end{gathered}
$$

Thus it is immediate that

$$
\begin{aligned}
J \leq & Q_{1, \xi \xi}-\theta\left(Q_{1} \frac{Z_{\xi}}{Z}\right)_{\xi} \\
+ & {\left[\alpha t^{-2 \alpha-1} Q_{0}+\alpha t^{-2 \alpha-1} \xi\left|\frac{\partial Q_{0}}{\partial \xi}\right|+\theta t^{-2 \alpha-1} \frac{Z^{\theta-1}}{\Gamma^{2}(t)} Q_{0}+\mu t^{-2 \alpha} Q_{0}+t^{-2 \alpha}\left|Q_{0, t}\right|\right] } \\
& +\left[\alpha t^{-2 \alpha-1} Q_{1}+\alpha t^{-2 \alpha-1} \xi\left|\frac{\partial Q_{1}}{\partial \xi}\right|+\theta t^{-2 \alpha-1} \frac{Z^{\theta-1}}{\Gamma^{2}(t)} Q_{10}+\mu t^{-2 \alpha} Q_{1}+t^{-2 \alpha}\left|Q_{1, t}\right|\right] .
\end{aligned}
$$

Using the inequalities in (39), we have

$$
\begin{aligned}
J & \leq Q_{1, \xi \xi}-\theta\left(Q_{1} \frac{Z_{\xi}}{Z}\right)_{\xi} \\
& +2\left[\alpha t^{-2 \alpha-1} Q_{0}+\alpha t^{-2 \alpha-1} \xi\left|\frac{\partial Q_{0}}{\partial \xi}\right|+\theta t^{-2 \alpha-1} \frac{Z^{\theta-1}}{\Gamma^{2}(t)} Q_{0}+\mu t^{-2 \alpha} Q_{0}+t^{-2 \alpha}\left|Q_{0, t}\right|\right] .
\end{aligned}
$$


With (38), we obtain $J \leq 0$, thus (36) follows.

Now we need to verify (39). We decompose $Q_{1}$ as a sum of $Q_{1,1}$ and $Q_{1,2}$, which solve

$$
\begin{gathered}
Q_{1,1, \xi \xi}-\theta\left(Q_{1,1} \frac{Z_{\xi}}{Z}\right)_{\xi}=-2\left[\alpha t^{-2 \alpha-1} Q_{0}+\alpha t^{-2 \alpha-1} \xi\left|\frac{\partial Q_{0}}{\partial \xi}\right|+\theta t^{-2 \alpha-1} \frac{Z^{\theta-1}}{\Gamma^{2}(t)} Q_{0}+t^{-2 \alpha}\left|Q_{0, t}\right|\right] \\
Q_{1,2, \xi \xi}-\theta\left(Q_{1,2} \frac{Z_{\xi}}{Z}\right)_{\xi}=-2 \mu t^{-2 \alpha} Q_{0} \\
Q_{1,1}(0, t)=Q_{1,2}(0, t)=0 .
\end{gathered}
$$

We will focus just on $Q_{1,2}$ since the analysis of $Q_{1,1}$ is similar and understanding $Q_{1,2}$ is more important in order to judge the role of $\mu$. We are interested in obtaining a particular solution for this differential equation. Because the equation for $Q_{1,2}$ can be rewritten as $\left(Z^{\theta}\left(Z^{-\theta} Q_{1,2}\right)_{\xi}\right)_{\xi}=-2 \mu t^{-2 \alpha} Q_{0}$, after integration we choose

$$
\left(Z^{-\theta} Q_{1,2}\right)_{\xi}=2 \mu t^{-2 \alpha} Z^{-\theta} \int_{\xi}^{\delta(\bar{t})^{\left(\frac{\theta-1}{3-\theta}\right)}} Q_{0}(\eta, t) d \eta
$$

Using $Q_{1,2}(0, t)=0$, we have

$$
Q_{1,2}(\xi, t)=2 \mu t^{-2 \alpha}(Z(\xi, t))^{\theta} \int_{0}^{\xi} d \lambda\left[(Z(\lambda, t))^{-\theta} \int_{\lambda}^{\delta(\bar{t})^{\left(\frac{\theta-1}{3-\theta}\right)}} Q_{0}(\eta, t) d \eta\right] .
$$

We can now estimate the behavior of $Q_{1,2}(\xi, t)$ for $\xi \gg 1$ and see how we choose $\mu$. Using Assumption 3.2, it follows that $Q_{0}$ behaves like $\xi^{-\frac{2 \theta}{\theta-1}}$ for large $\xi$. It is then easy to see that $Q_{1,2}$, up to multiplicative constants, behaves like

$$
Q_{1,2} \approx \mu t^{-2 \alpha} \frac{1}{\xi^{\frac{2 \theta}{\theta-1}}} \xi^{2} \approx \mu t^{-2 \alpha} \xi^{-\frac{2}{\theta-1}}
$$

for large $\xi$, by recalling that $\alpha=\frac{\theta-1}{3-\theta}$. Now we can compare $Q_{0}$ with $Q_{1,2}$. Note that $Q_{0}>0$ for $|\xi| \approx \delta t^{\frac{\theta-1}{3-\theta}}$. Moreover

$$
Q_{0} \approx \xi^{-\frac{2 \theta}{\theta-1}}, \quad Q_{1,2} \approx \mu t^{-2 \alpha} \xi^{-\frac{2}{\theta-1}} .
$$

Therefore, for $\mu$ of order one and $\delta$ small it follows that $\left|Q_{1,2}\right| \ll Q_{0}$. In a similar manner, we can show that $\left|\left(Q_{1,2}\right)_{\xi}\right| \ll\left|\left(Q_{0}\right)_{\xi}\right|$ and $\left|\left(Q_{1,2}\right)_{t}\right| \ll\left|\left(Q_{0}\right)_{t}\right|$. Details are omitted. This completes the estimate (39). 
So, we conclude that $\mathcal{H}_{Z}\left(\mathcal{G}_{3}\right) \leq 0$, which yields $\mathcal{H}_{Z}(\mathcal{G}) \leq 0$. Indeed, together with (32), (35), we obtain

$$
\begin{gathered}
\mathcal{H}_{Z}\left(\mathcal{G}_{1}+\mathcal{G}_{2}+\mathcal{G}_{3}-G\right) \leq \mathcal{H}_{Z}\left(\mathcal{G}_{1}\right)+\mathcal{H}_{Z}\left(\mathcal{G}_{2}\right)-\mathcal{H}_{Z}(G) \\
\approx t^{-2(2 \alpha+1)}\left(K_{1}+1\right)\left(\xi^{2}+a\right)^{-\frac{1}{\theta-1}}+t^{-3(2 \alpha+1)}\left(\xi^{2}+a\right)^{-\frac{2-\theta}{\theta-1}} \\
+t^{-2 \alpha-1} \gamma(t) \frac{1+K_{2}}{\left(\xi^{2}+a\right)^{\frac{\theta}{\theta-1}}}+t^{-4 \alpha-2} \gamma(t)\left(\frac{1}{\left(\xi^{2}+a\right)^{\frac{\theta}{\theta-1}}}+\frac{1}{\left(\xi^{2}+a\right)^{\frac{1}{\theta-1}}}\right),
\end{gathered}
$$

where we used that $\mathcal{H}_{Z}(\mathcal{G})=t^{-2 \alpha-1} \mathcal{R}_{1}$. Suppose that $t$ is as large as needed, which can be obtained by setting $\bar{t} \geq t_{0}$ for an arbitrary large number $t_{0}$. By choosing constants $K_{1}$ and $K_{2}$ such that $K_{1}+1<0$ and $K_{2}+1<0$, we can obtain that $\mathcal{H}_{Z}\left(\mathcal{G}_{1}+\mathcal{G}_{2}+\mathcal{G}_{3}-G\right)<0$.

In order to apply the maximum principle, we need $\mathcal{G}_{1}+\mathcal{G}_{2}+\mathcal{G}_{3}>G$ for $t=\bar{t}$ and $G \geq C t^{-\frac{3(\theta+1)}{2(3-\theta)}}$ for $|\xi|=\delta t^{\alpha}$.

The positivity of $\mathcal{G}_{1}+\mathcal{G}_{2}+\mathcal{G}_{3}-G$ for $t=\bar{t}$ is due to the fact that $\mathcal{G}_{3}$ is the largest term among $\left\{\mathcal{G}_{i}: i=1,2,3\right\}$ and $\mathcal{G}_{3}>G$ for $t=\bar{t}$. On the other hand, at the boundary $|\xi|=\delta t^{\alpha}$ the inequality $G \geq C t^{-\frac{3(\theta+1)}{2(3-\theta)}}$ results from the fact that $\mathcal{G}_{3}>0$ and $\mathcal{G}_{2}>C t^{-\frac{3(\theta+1)}{2(3-\theta)}}$ for $|\xi|=\delta t^{\alpha}$. Note that $\mathcal{G}_{1}$ is added to control the "small nonlinear terms", which are very small compared with $\mathcal{G}_{2}$. Summing up all above given, we conclude that $\mathcal{G}_{1}+\mathcal{G}_{2}+\mathcal{G}_{3} \geq G$ is a super-solution for $G$.

With this construction of a super-solution above, we can now prove Theorem 3.9.

Proof of Lemma 3.9 : Since the super-solution given above is bounded by $C t^{-\frac{1}{2}} Z^{\theta}(\xi, t)$ for large $t$ with $t \geq \bar{t}+K \log (\bar{t})$, it follows that there exists $\beta>0$ such that the supersolution is bounded by $C t^{-\beta} Z^{\theta}(\xi, t)$ for any $t \geq t_{0}$, and thus, back in the original variable, we obtain

$$
\left|u(x, t)-\frac{z^{\theta}}{\int_{I} z^{\theta} d x}\right|=t^{\frac{\theta-1}{3-\theta}}|G(\xi, t)| \leq C t^{-\beta} t^{\frac{\theta-1}{3-\theta}} \frac{Z^{\theta}(\xi, t)}{\int_{I} Z^{\theta}(\xi, t) d \xi}=C t^{-\beta} \frac{z^{\theta}(x, t)}{\int_{I} z^{\theta}(x, t) d x},
$$

where we used $\int_{I} Z^{\theta}(\xi, t) d \xi \leq C$, with $C=C(\theta, M)$ depends on. This completes the proof of our lemma.

Finally we conclude the proof of the main theorem in this section with

Lemma 3.11 There exist solutions $u, z$ which satisfy all conditions of Assumption 3.2 for all $t \geq t_{0}$.

Proof. Without loss of generality, the initial time of our problem is $t_{0}$, since the system under consideration is invariant under time translations $t \rightarrow t-t_{0}$. Our choice of initial data $u\left(\cdot, t_{0}\right)$ and $z\left(\cdot, t_{0}\right)$ is sufficiently smooth and moreover, $u\left(\cdot, t_{0}\right)$ is assumed to be very close to the expected asymptotic behavior $t^{\frac{\theta-1}{3-\theta}}\left(B x^{2} t^{\frac{2(\theta-1)}{3-\theta}}+A\right)^{-\frac{1}{\theta-1}}$. 
We begin proving the $L^{\infty}$-estimate for $z$ according to Assumption 3.2, 2. By Lemma 3.9 we have

$$
\left|z_{t}-\frac{z^{\theta}}{\int_{I} z^{\theta} d x}\right| \leq \varepsilon(t) \frac{z^{\theta}}{\int_{I} z^{\theta} d x}
$$

Integrating this equation starting from $t=t_{0}$, exactly as we did in the formal computations at the begining of Section 3 we obtain 2. in Assumption 3.2, which implies that $z$ blows up in infinite time. More precisely, if for the initial data we have

$$
\left|z_{0}\left(x, t_{0}\right)-\frac{t_{0}^{\frac{2}{3-\theta}}}{\left(B x^{2} t_{0}^{\frac{2(\theta-1)}{3-\theta}}+A\right)^{\frac{1}{\theta-1}}}\right| \leq \frac{1}{2} \frac{t_{0}^{\frac{2}{3-\theta}}}{\left(B x^{2} t_{0}^{\frac{2(\theta-1)}{3-\theta}}+A\right)^{\frac{1}{\theta-1}}} .
$$

Therefore we recover 2. in Assumption 3.2 with $M$ replaced by $\frac{M}{2}$ for $t_{0} \leq t<\infty$, if $M$ is sufficiently large and then $t_{0}$ chosen accordingly large enough.

Finally we derive the estimates for the derivatives and the Hölder norms in 1.,3., and 4. of Assumption 3.2. The arguments are similar as those given in [2] and [3]. Suppose that $\bar{t} \geq t_{0}$. We introduce a characteristic length $R$ satisfying $\frac{1}{(\bar{t})^{\left(\frac{2(\theta-1)}{3-\theta}\right)}} \leq R<1$. Using the new variables $y=x / R$ and $\tau=(t-\bar{t}) / R^{2}$, we define

$$
v_{R}(y, \tau):=R^{\frac{2 \theta}{\theta-1}} v\left(R y, \bar{t}+R^{2} \tau\right), \quad z_{R}(y, \tau):=R^{\frac{2}{\theta-1}} z\left(R y, \bar{t}+R^{2} \tau\right) .
$$

Then, for $\tau \in(0,1)$ and $|y| \in(1 / 2,3 / 2)$, we have

$$
\left|v_{R}(y, \tau)\right| \leq \frac{2 \epsilon(\bar{t})}{(\bar{t})^{\frac{\theta+1}{3-\theta}}},\left|z_{R}(y, \tau)\right| \leq 2 .
$$

On the other hand $v_{R}$ and $z_{R}$ satisfy

$$
\begin{gathered}
v_{R, \tau}=v_{R, y y}-\theta\left(\frac{z_{R, y}}{z_{R}} v_{R}\right)_{y}-\frac{1}{R^{2}}\left(\frac{z_{R}^{\theta}}{\alpha(\tau)}\right)_{\tau}, \\
z_{R, \tau}=\frac{z_{R}^{\theta}}{\alpha(\tau)}+v_{R},
\end{gathered}
$$

with

$$
\alpha(\tau)=\int_{I} z^{\theta} d x \approx(\bar{t})^{\left(\frac{\theta+1}{3-\theta}\right)} .
$$

The term $\left(\frac{z_{R}^{\theta}}{\alpha(\tau)}\right)_{\tau}$ can be shown to be sufficiently smooth and small by using (41), since

$$
\left(\frac{z_{R}^{\theta}}{\alpha(\tau)}\right)_{\tau}=\frac{\theta z_{R}^{\theta-1}}{\alpha(\tau)}\left(\frac{z_{R}^{\theta}}{\alpha(\tau)}+v_{R}\right)-\frac{\alpha^{\prime}(\tau)}{\alpha^{2}(\tau)} z_{R}^{\theta}
$$


Note that $\alpha^{\prime}(\tau) \approx(\bar{t})^{\frac{2(\theta-1)}{3-\theta}}$. One can easily see that the above terms are small contributions compared to other terms on the right hand side of (40). In order to obtain $\mathcal{C}^{2+\nu}$ estimates, we first take two spatial derivatives in (41) and obtain

$$
\left(\frac{\partial^{2} z_{R}}{\partial y^{2}}\right)_{\tau}=\frac{\theta(\theta-1) z_{R}^{\theta-2}}{\alpha(\tau)}\left(\frac{\partial z_{R}}{\partial y}\right)^{2}+\frac{\theta z_{R}^{\theta-1}}{\alpha(\tau)} \frac{\partial^{2} z_{R}}{\partial y^{2}}+\frac{\partial^{2} v_{R}}{\partial y^{2}}
$$

The above equation indicates that we have to control $\frac{\partial^{2} v_{R}}{\partial y^{2}}$. In fact, an interior regularity result for $v_{R}$ in the region $\frac{3}{4} \leq|y| \leq \frac{5}{4}$ is needed. We introduce a cutoff $\xi(y)$ which equals 1 for $\frac{3}{4} \leq|y| \leq \frac{5}{4}$ and vanishes for $|y-1|>\frac{1}{2}$. Then for $\xi(y) v_{R}=: \bar{v}_{R}$ it follows

$$
\bar{v}_{R, \tau}=\bar{v}_{R, y y}-\theta\left(\frac{z_{R, y}}{z_{R}} \bar{v}_{R}\right)_{y}-2 v_{R, y} \xi_{y}-v_{R} \xi_{y y}+\theta\left(\frac{z_{R, y}}{z_{R}} v_{R} \xi_{y}\right)-\frac{1}{R^{2}}\left(\frac{z_{R}^{\theta} \xi}{\alpha(\tau)}\right)_{\tau} .
$$

The equation for $\bar{v}_{R}$ is similar to the one for $v_{R}$ except for some source terms that are of order $\frac{\epsilon(\bar{t})}{\bar{t}^{\frac{\theta+1}{3}-\theta}}$. Since $\bar{v}_{R}$ vanishes, it follows that as long as $z_{R}$ satisfies Assumption 3.1, the fundamental solution of the equation satisfied by $\bar{v}_{R}$ decreases exponentially in $\tau$, and the $C^{2, \nu}$-derivatives in space also decay exponentially by standard regularizing effects. More precisely, we obtain two types of contributions for the derivatives of $\bar{v}_{R}$, one of which is the part associated to the initial data starting at $t=\bar{t}$ that decreases exponentially, and a second part associated to the source term which is of order $\frac{\epsilon(\bar{t})}{(\bar{t})^{\frac{\theta+1}{3-\theta}}}$. Due to the decay of the function $\frac{1}{\alpha(\tau)}$, we can obtain a similar decay for the derivatives of $z_{R}$ and the Hölder estimates, by using derivatives of $v_{R}$ as source terms in the equation (41). This gives the desired estimate for any $t \geq 2$. If $t \leq 2$ we obtain similar results for $\left\|v_{R}(t)\right\|_{C^{2, \nu}},\left\|z_{R}\right\|_{C^{2, \nu}}$ using the regularity of the initial data $v_{0}(x), z_{0}(x)$. In particular for $t \in[\bar{t}, \bar{t}+1]$ we can derive

$$
\begin{aligned}
\left\|v_{R}(\bar{t}+1)\right\|_{\mathcal{C}^{2, \nu}} & \leq \sigma\left\|v_{R}(\bar{t})\right\|_{\mathcal{C}^{2, \nu}}+\frac{C \epsilon(\bar{t})}{(\bar{t})^{\frac{\theta+1}{3-\theta}}}, \\
\left\|z_{R}(\bar{t}+1)\right\|_{\mathcal{C}^{2, \nu}} & \leq \sigma\left\|z_{R}(\bar{t})\right\|_{\mathcal{C}^{2, \nu}}+C \epsilon(\bar{t}),
\end{aligned}
$$

where $0<\sigma<1$ due to the exponential decay of the solutions for the initial data mentioned above. The main contribution is due to the sources. Usual iterative methods yield the global smallness estimates as desired. Taking the supremum for all the admissible values of $R$ and returning to the original variables $(x, t)$, we obtain estimates for the Hölder norms defined in Assumption 3.2. This completes the proof of our main theorem.

\section{Acknowledgement:}

This paper was done while all three authors were at the Max-Planck-Institute for Mathematics in the Sciences, in Leipzig, Germany. Additionally the first author was partially supported by KRF-2006-331-C00020. The third author was also supported by the Humboldt foundation and by DGES Grant MTM2007-61755. Further support was given by The International Graduiertenkolleg IGK 710, Heidelberg, funded by the Deutsche Forschungsgemeinschaft, (DFG). 


\section{References}

[1] R. Courant \& D. Hilbert Methods of Mathematical Physics. Reprint of the 1962 original. Wiley Classics Library (1989), Wiley \& Sons, New York.

[2] M.A. Herrero \& J.J.L. Velazquez Singularity patterns in a chemotaxis model. Math. Ann. 306 (1996), 583-623.

[3] M.A. Herrero \& J.J.L. Velazquez A blow-up mechanism for a chemotaxis model. Ann. Scuola Norm. Sup. Pisa XXIV (1997), 1739-1754.

[4] T. Horiuchi Best constant in weighted Sobolev inequality with weights being powers of distance from the origin, J. of Inequal. \& Appl. 1 (1997), no. 3, 275-292.

[5] E. Keller \& L. Segel Traveling bands of chemotactic bacteria:A theoretical analysis, J. Theor. Biol. 30 (1971), 235-248.

[6] H. Levine \& B. Sleeman A system of reaction diffusion equations arising in the theory of reinforced random walks. SIAM J. Appl. Math 57 (1997), no. 3, 683-730.

[7] V.G. MaZ'JA Sobolev spaces (1985), Springer Verlag, Berlin.

[8] H. G. Othmer \& A. Stevens Aggregation, blowup, and collapse: the ABC's of taxis in reinforced random walks. SIAM J. Appl. Math. 57 (1997), no. 3, 1044-1081.

[9] M. RASCLE Sur uné equation intégro-différentielle non lineaire issue de la biologie, J. Diff. Equ. 32 (1979), no. 3, 420-453.

[10] H. SchwetLick, personal communication.

[11] A. Stevens Trail following and aggregation of myxobacteria. J. of Biol. Systems 3 (1995), 1059-1068.

[12] M. Struwe Variational methods. Applications to nonlinear differential equations and Hamiltonian systems, (2000), Springer Verlag, Berlin.

[13] Y. YANG, H. Chen \& W. LiU On existence of global solutions and blow-up to a system of reaction diffusion equations modelling chemotaxis, SIAM J. Math. Anal. 33 (2001), no. 4, 763-785. 